\title{
Effects of continuous positive airway pressure on energy balance regulation: a systematic review
}

\author{
Ari Shechter
}

Affiliation:

Department of Medicine, Columbia University, New York, NY, USA.

\section{Correspondence:}

Ari Shechter, 1150 St Nicholas Ave, Room 121, New York, NY, 10032, USA.

E-mail: as4874هcolumbia.edu

ABSTRACT Obesity is both a cause and a possible consequence of obstructive sleep apnoea (OSA), as OSA seems to affect parameters involved in energy balance regulation, including food intake, hormonal regulation of hunger/satiety, energy metabolism and physical activity. It is known that weight loss improves OSA, yet it remains unclear why continuous positive airway pressure (CPAP) often results in weight gain.

The goal of this systematic review is to explore if and how CPAP affects the behaviour and/or metabolism involved in regulating energy balance.

CPAP appears to correct for a hormonal profile characterised by abnormally high leptin and ghrelin levels in OSA, by reducing the circulating levels of each. This is expected to reduce excess food intake. However, reliable measures of food intake are lacking, and not yet sufficient to make conclusions. Although studies are limited and inconsistent, CPAP may alter energy metabolism, with reports of reductions in resting metabolic rate or sleeping metabolic rate. CPAP appears to not have an appreciable effect on altering physical activity levels. More work is needed to characterise how CPAP affects energy balance regulation.

It is clear that promoting CPAP in conjunction with other weight loss approaches should be used to encourage optimal outcomes in OSA patients.

@ERSpublications

CPAP in sleep apnoea patients affects some aspects of energy balance regulation, which can affect body weight http://ow.ly/6ATZ303KXRv 


\section{Introduction}

Obesity is among the leading risk factors for obstructive sleep apnoea (OSA), and percentage body fat, visceral adiposity and body mass index (BMI) are found to be significantly related to the apnoea-hypopnoea index (AHI) [1-4]. OSA and obesity appear to exist in a reciprocal relationship, whereby OSA plays a role in promoting further weight gain [5]. Considering the effects of sleep disruption on factors regulating body weight, namely food intake, the hormonal regulation of hunger/satiety, energy metabolism and physical activity, it is expected that the arousals, sleep fragmentation and disrupted sleep architecture that characterise OSA will be associated with behavioural, metabolic and/or hormonal changes favouring weight gain [6].

There is some evidence for a dysregulation of hunger/satiety-regulating hormones in OSA, with increased leptin levels, suggestive of leptin resistance [7-16], and increased ghrelin [9, 17-19] relative to controls. OSA is associated with a hormonal profile that may encourage low satiety (via leptin resistance) and high hunger (via ghrelin) and therefore excessive energy intake. Although food intake has rarely been studied in OSA, in children AHI is positively associated with the caloric, fat and carbohydrate content of a self-selected meal [20], and in adults, liking for high-fat food was associated with greater OSA severity [21]. In terms of energy expenditure, OSA appears to be characterised by lower levels of physical activity, as assessed subjectively by questionnaire [22] and objectively using accelerometry [23]. In addition, OSA may affect energy metabolism, with reports of elevated resting metabolic rate (RMR) [24], sleeping energy expenditure [25] and 24-h energy expenditure in patients versus controls [26].

It appears that OSA does have an effect of altering energy balance, i.e. the quantifiable relationship between energy intake and energy expenditure, in such a way as to promote weight gain. An increased understanding of how OSA and its treatment affect energy balance is important, as it can help improve body weight management in patients. For instance, it is known that body weight changes are related to OSA, as a $10 \%$ increase in weight predicted a $32 \%$ increase in AHI and similar weight loss predicted a $26 \%$ decrease in AHI over 4 years of follow-up [27]. Randomised controlled trials (RCTs) consisting of reduced caloric intake $[28,29]$ or combined reduced caloric intake and prescribed physical activity [30] demonstrated the effectiveness of behavioural lifestyle interventions which induce weight loss to improve OSA. The first-line treatment for OSA is continuous positive airway pressure (CPAP). However, whether CPAP treatment affects body weight in OSA patients is controversial. Weight loss has been shown in some $[31,32]$, but not all CPAP intervention studies [33, 34], and some investigations suggest that CPAP use promotes weight gain $[35,36]$. A recent meta-analysis of 25 controlled trials $(n=3181$ patients) reported that CPAP results in a significant increase in body weight, although the magnitude of weight gain was relatively small (Hedges' $\mathrm{d}=0.17$ ) [37]. The reasons remain unclear, but probably involve the aforementioned energy balance-related factors.

The current goal is to explore whether and how CPAP affects behaviour and/or metabolism involved in regulating energy balance, including energy intake and energy expenditure. This review focuses on observational and laboratory-based studies that investigated outcomes such as food intake, the hormonal regulation of hunger/satiety, physical activity and energy metabolism in OSA patients using CPAP, to determine if CPAP affects these energy balance-related parameters.

\section{Methods}

Search strategy

Articles that investigated energy intake and/or energy expenditure in OSA patients in response to CPAP were identified using a systematic search of the published literature. Web-based searches were conducted for manuscripts in the PubMed/Medline, Embase and Cochrane Library databases. Specific search terms reflect the treatment/intervention (CPAP) and outcomes (energy balance-related parameters). For treatment/ intervention, search terms included CPAP or positive airway pressure. For energy intake outcomes, search terms included caloric intake; food intake; food preference; dietary quality; macronutrient; hunger; appetite; satiety; hunger hormone; appetite hormone; and satiety hormone. For energy expenditure outcomes, search terms included energy expenditure; thermogenesis; energy metabolism; and physical activity. Key terms were searched in all possible combinations using Boolean logic operators. A manual search of all bibliographies of the included articles was conducted to identify relevant references missed by automated searches. EndNote X7 (Thomson Reuters, New York, NY, USA) was used to index and manage all references.

\section{Eligibility criteria}

Specific inclusion criteria were required for selection. The criteria included: 1) original research investigations; 2) conducted in humans; 3) conducted in adults; 4) include patients diagnosed with OSA of at least mild severity; 5) include administration of CPAP for $\geqslant 1$ day; and 6) include at least one energy balance-related outcome (food intake, hunger/appetite, energy expenditure, energy metabolism or physical activity). Studies were excluded if they were only comparisons between OSA patients and non-OSA 
controls and/or were limited to untreated OSA patients. Design of CPAP studies could be pre-versus post-treatment or placebo-controlled trials. No restriction on length of follow-up for CPAP treatment was included (range 1 day to 11 months). If a study contained CPAP treatment combined with a lifestyle intervention of prescribed low-calorie diet and physical activity recommendations, only the control CPAP group with no lifestyle intervention was included [38], as the lifestyle intervention would necessarily affect primary outcomes.

No date limitations or restrictions on country of research or publication were imposed in an effort to reduce source selection bias. To be included, specified energy balance-related parameters could be secondary or minor outcomes within the reports. Reviews, editorials and commentaries were excluded. Conference abstracts were included, but were excluded from the final synthesis if no data were presented [39-42], if they presented data that were subsequently presented in a full manuscript otherwise included [43-45] or if the methodology was not adequately described [46]. In addition to a "forward" (utilising databases and search terms) search strategy, a "backward" or ancestry search strategy was used, such that the bibliography of all relevant reports was searched for references missed by web-based/database searches.

\section{Data items}

To be selected, studies must have explored at least one relevant energy balance-related parameter in OSA patients after CPAP treatment. For energy intake, this included circulating hormones known to regulate hunger, satiety or appetite (leptin, ghrelin, etc.), and measures of food intake, habitual dietary patterns (test meals, food diaries or dietary preference scales) or eating behaviours. For energy expenditure, this included measures from indirect calorimetry (resting/basal metabolic rate, sleeping metabolic rate or 24-h energy expenditure), accelerometry (step counts or metabolic equivalent units) and questionnaires on physical activity levels. Data on changes in body weight and/or composition after CPAP were not included, and are the topic of a recent meta-analysis [37].

\section{Reviewing procedure and data extraction}

Database searches were conducted in March 2016. All obtained references were reviewed, and if retained, data extraction was conducted by a single author. The first level of review was title and abstract screening. Irrelevant references were removed. Potentially relevant studies were further assessed for inclusion by reading the full text and checking against pre-specified eligibility criteria.

For each reference, the following variables were systematically extracted and entered into a summary table: 1) authors; 2) publication year; 3) journal; 4) criteria and cut-offs used to define OSA; 5) sample size; 6) baseline age, BMI and Epworth sleepiness scale (ESS) score; 7) study design; 8) duration of CPAP use in the treatment; 9) CPAP compliance (duration of use during treatment phase); 10) outcome variables assessed, including time of assessment and if performed under fasting conditions; and 12) main findings. The principal summary measures were differences in means for energy balance-related outcomes. Studies included consisted of pre-versus post-CPAP comparisons (baseline to post-treatment) and placebocontrolled trials (active versus sham CPAP or CPAP versus another non-CPAP control).

\section{Results}

\section{Search results and selection of references}

A summary of the numbers of studies screened, assessed and included is presented in figure 1. After screening and assessment, 42 references were included in the final synthesis. Studies were classified based on their primary outcomes: one study on food intake, 22 studies on hormones affecting food intake, six studies on energy expenditure assessed via indirect calorimetry and 13 studies on physical activity. Several studies also described relevant secondary outcomes, and therefore had data that were included in other categories. Specifically, the one study included in the energy intake category also contained data on physical activity [47]. One of the studies in the indirect calorimetry category also contained data on energy intake, hormones and physical activity [48]. Two studies in the physical activity category also contained data on energy intake $[38,49]$.

\section{Food intake}

To date, four studies have investigated the effects of CPAP on energy intake or food preference either as primary [47] or secondary outcomes $[38,48,49]$ (table 1). A large group of participants with AHI $\geqslant 10$ events. $h^{-1}(n=230)$ were randomised to sham or active CPAP as part of the Apnea Positive Pressure Long-term Efficacy Study (APPLES) [47]. Active versus sham groups in this RCT were matched for sex, BMI and ESS, and participants completed the food frequency questionnaire (FFQ) to assess typical food intake after treatment. In the 4-month follow-up to baseline assessment, the only significant effect of active CPAP was a reduction in trans-fatty acid consumption in women [47]. It should be noted that after randomisation, men in both groups had lower BMIs than women. Another RCT in which participants either received a 


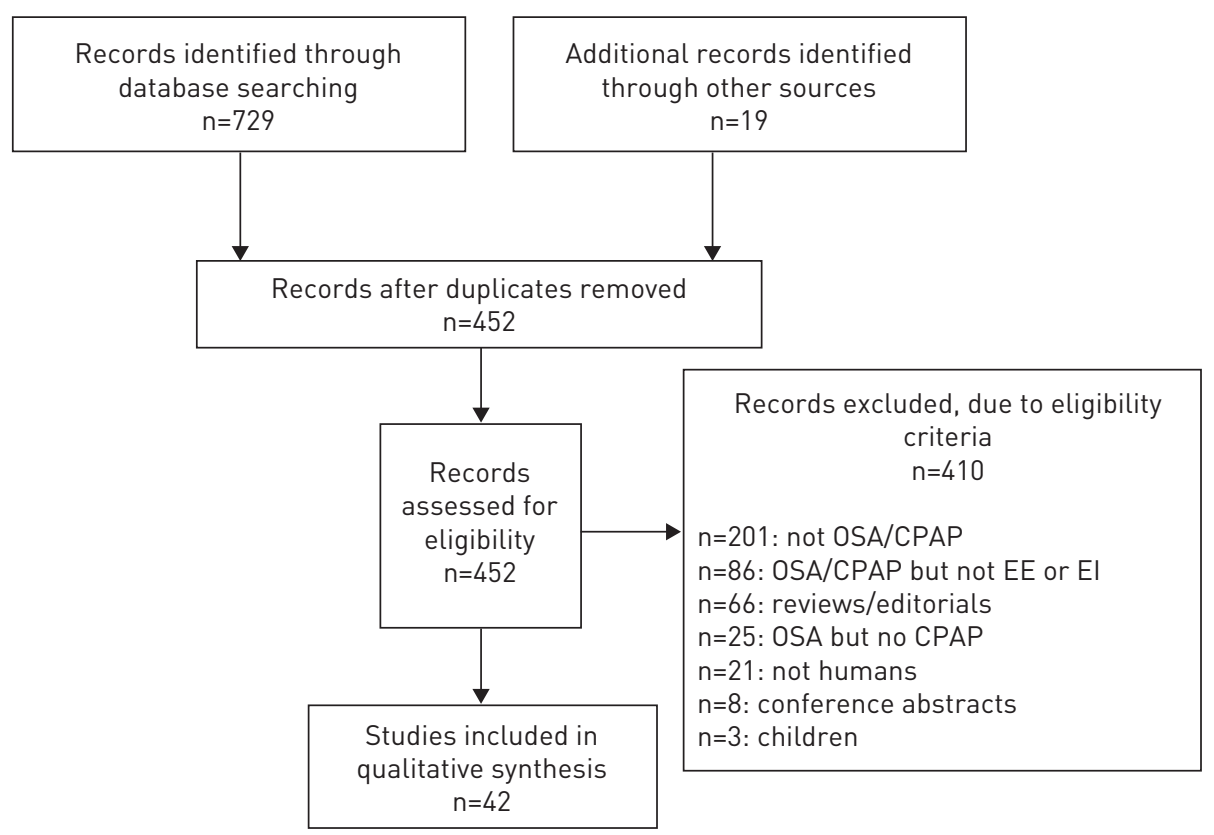

FIGURE 1 Flow chart for the selection of references included in the systematic literature review. OSA: obstructive sleep apnoea; CPAP: continuous positive airway pressure; EE: energy expenditure; El: energy intake.

confirmed 8-h duration of nightly CPAP for 2 weeks, or an oral placebo, reported no between-group differences in self-reported food intake by daily meal log [49]. In a third RCT, participants either received CPAP combined with behavioural lifestyle intervention or just CPAP as control [38]. Focusing on the control group, CPAP did not affect consumption of vegetables, fruits/berries, fish/shellfish or sweets from baseline to the 6-month follow-up, as assessed using an eating habits questionnaire [38]. In that study, after 6 months of treatment the control group had a reduced score on the emotional eating component of the Dutch eating behaviour questionnaire, indicating that these participants reduced their emotional approach to eating [38]. Finally, dietary habits were assessed using a self-administered diet history questionnaire at baseline and after 3 months of treatment [48]. No changes in energy intake were observed for the entire group. However, when stratified by changes in body weight after treatment, those who gained weight had a significantly higher total caloric intake at follow-up versus those who did not gain weight.

\section{Hormonal regulation of food intake}

Findings on the effects of CPAP on the hormonal regulation of food intake are summarised in table 2. Several within-patient studies have investigated the effects of CPAP on leptin levels by comparing baseline to post-treatment values, with 12 reporting significant reductions in leptin after treatment $[7,9,10,32,33$, 50-56] and six reporting no change [17, 18, 48, 57-59]. In most cases, leptin was sampled in the morning after an overnight fast $[7,9,10,17,18,32,33,48,50-52,55-59]$, and in one case, which did report post-treatment reductions, it was sampled throughout the night [53]. The duration of treatment from baseline to follow-up ranged from 1 day to 6 months, and does not seem to contribute to disparate findings. Importantly, it appears that only two studies to date have utilised placebo-controlled RCT designs, and reported no change in leptin levels between active and placebo CPAP conditions after 2 [60] or 3 months [61] of treatment.

Five within-patient studies investigated plasma ghrelin levels at baseline and after CPAP. Four of these reported a significant reduction in ghrelin levels after treatment duration of 2 days [9], 1 month [18], 3 months [17] and 6 months [58]. In the study with the 1-month follow-up, despite significantly reduced levels at the end-point, ghrelin levels were not changed at a proximal follow-up after 3-4 days of treatment [18]. One study observed no change in ghrelin after treatment of 1 day or 3 months [48]. Each of the aforementioned studies sampled ghrelin levels in the morning in fasted conditions. The one study with negative results had similar CPAP compliance $(\sim 4.5 \mathrm{~h}$ per night $)$ as the investigations showing a reduction in ghrelin, so nightly CPAP use is unlikely to contribute to the inconsistency.

Findings on the effects of CPAP on orexin (a hypothalamic neuropeptide which increases food intake) are inconsistent, owing mainly to the small number of studies, which varied in design [62-64]. In a within-subject pre-post comparison, treatment with CPAP of 3-4 months was found to reduce plasma 
TABLE 1 Summary of effects of continuous positive airway pressure (CPAP) on food intake in obstructive sleep apnoea (OSA) patients

\begin{tabular}{|c|c|c|c|c|c|c|c|c|c|c|c|c|}
\hline First author [ref.] & Year & Participants & $\begin{array}{l}\text { AHI cut-off } \\
\text { events } \cdot h^{-1}\end{array}$ & $\begin{array}{l}\text { Sample } \\
\text { size }\end{array}$ & $\begin{array}{c}\text { Age } \\
\text { years } M / F\end{array}$ & $\begin{array}{l}\text { Baseline BMI } \\
\mathrm{kg} \cdot \mathrm{m}^{-2} \mathrm{M} / \mathrm{F}\end{array}$ & $\begin{array}{l}\text { Baseline } \\
\text { ESS M/F }\end{array}$ & Design & $\begin{array}{l}\text { CPAP } \\
\text { duration }\end{array}$ & $\begin{array}{c}\text { CPAP } \\
\text { compliance } h \text { per } \\
\text { night } M / F\end{array}$ & Outcomes & $\begin{array}{l}\text { Main findings: } \\
\text { effect of CPAP }\end{array}$ \\
\hline Batool-Anwar [47] & 2014 & $\begin{array}{l}\text { Active CPAP } \\
\text { Sham CPAP }\end{array}$ & $\begin{array}{l}\geqslant 10 \\
\geqslant 10\end{array}$ & $\begin{array}{l}117 \\
114\end{array}$ & $\begin{array}{c}54.0 \pm 13.0 / \\
55.0 \pm 13.0 \\
55.0 \pm 14.0 / \\
54.0 \pm 13.0\end{array}$ & $\begin{array}{c}33.0 \pm 6.3^{*} / \\
36.0 \pm 11.0 \\
31.0 \pm 5.5 * / \\
34.0 \pm 8.8\end{array}$ & $\begin{array}{c}10.0 \pm 4.0 / \\
10.7 \pm 4.6 \\
9.7 \pm 4.3 / \\
9.7 \pm 3.7\end{array}$ & $\begin{array}{l}\text { Sham-controlled, } \\
\text { parallel; RCT }\end{array}$ & $\begin{array}{l}4 \text { months } \\
4 \text { months }\end{array}$ & $\begin{array}{c}4.0 \pm 2.9 * * / \\
3.5 \pm 2.8 \\
2.6 \pm 2.6 * * / 2.9 \pm 2.6\end{array}$ & $\begin{array}{c}\text { Food } \\
\text { frequency } \\
\text { questionnaire }\end{array}$ & $\begin{array}{c}\downarrow \text { servings of } \\
\text { trans-fatty acids in } \\
\text { women after } \\
\text { treatment }\end{array}$ \\
\hline IGELSTROM [38] & 2014 & OSA & $\geqslant 15$ & 35 & $53.0 \pm 11.0$ & $33.6 \pm 4.3$ & $N / R$ & $\begin{array}{c}\text { Baseline } \\
\text { to post-treatment; } \\
\text { RCT }\end{array}$ & 6 months & $\begin{array}{l}>4 \text { in } 73 \% \text { of } \\
\text { participants }\end{array}$ & $\begin{array}{c}\text { Food intake } \\
\text { questionnaire } \\
\text { Dutch eating } \\
\text { behaviour } \\
\text { questionnaire }\end{array}$ & $\begin{array}{c}\leftrightarrow \text { food intake } \\
\text { patterns } \\
\downarrow \text { emotional } \\
\text { eating score after } \\
\text { treatment }\end{array}$ \\
\hline \multirow[t]{2}{*}{ PAMIDI [49] } & 2015 & CPAP & $\geqslant 5$ & 26 & $53.8 \pm 6.2$ & $36.8 \pm 7.8$ & $10.0 \pm 5.9$ & \multirow[t]{2}{*}{$\begin{array}{l}\text { Placebo-controlled, } \\
\text { parallel; RCT }\end{array}$} & 2 weeks & 8 & \multirow[t]{2}{*}{$\begin{array}{l}\text { Daily food } \\
\quad \log \end{array}$} & \multirow{2}{*}{$\begin{array}{c}\leftrightarrow \text { in food intake } \\
\text { between groups } \\
\text { after treatment }\end{array}$} \\
\hline & & $\begin{array}{l}\text { Oral } \\
\text { placebo }\end{array}$ & $\geqslant 5$ & 13 & $55.2 \pm 8.4$ & $32.7 \pm 4.3$ & $10.9 \pm 5.0$ & & 2 weeks & & & \\
\hline TACHIKAWA [48] & 2016 & OSA & $>20$ & 63 & $60.6 \pm 10.0$ & $27.9 \pm 3.8$ & $8.7 \pm 5.3$ & $\begin{array}{l}\text { Baseline to } \\
\text { post-treatment }\end{array}$ & $\begin{array}{c}1 \text { day; } \\
3 \text { months }\end{array}$ & $4.5 \pm 1.6$ & $\begin{array}{l}\text { Diet history } \\
\text { questionnaire }\end{array}$ & $\begin{array}{l}\leftrightarrow \text { energy intake } \\
\text { after treatment for } \\
\text { entire group } \\
\uparrow \text { energy intake } \\
\text { after treatment in } \\
\text { weight gainers }\end{array}$ \\
\hline
\end{tabular}

Data are presented as $\mathrm{n}$ or mean $\pm \mathrm{SD}$, unless otherwise stated. AHI: apnoea-hypopnoea index; M: male; F: female; BMI: body mass index; ESS: Epworth sleepiness scale; RCT: randomised controlled trial; N/R: not reported. $\uparrow$ : significant increase; $\downarrow$ : significant decrease; $\leftrightarrow$ : no difference. *: $p<0.05 \mathrm{M}$ versus $\mathrm{F}^{* *}$ : $p<0.05$ versus sham within males. 


\begin{tabular}{|c|c|c|c|c|c|c|c|c|c|c|c|c|}
\hline First author [ref.] & Year & Participants & $\begin{array}{l}\text { AHI cut-off } \\
\text { events } \cdot h^{-1}\end{array}$ & $\begin{array}{l}\text { Sample } \\
\text { size }\end{array}$ & $\begin{array}{c}\text { Age } \\
\text { years } M / F\end{array}$ & $\begin{array}{c}\text { Baseline BMI } \\
\mathrm{kg} \cdot \mathrm{m}^{-2} \mathrm{M} / \mathrm{F}\end{array}$ & $\begin{array}{l}\text { Baseline } \\
\text { ESS M/F }\end{array}$ & Design & $\begin{array}{l}\text { CPAP } \\
\text { duration }\end{array}$ & $\begin{array}{c}\text { CPAP } \\
\text { compliance } h \text { per } \\
\text { night } M / F\end{array}$ & Outcomes & $\begin{array}{l}\text { Main findings: } \\
\text { effect of CPAP }\end{array}$ \\
\hline \multicolumn{13}{|l|}{ Leptin } \\
\hline Barcelo [7] & 2005 & $\begin{array}{l}\text { OSA obese } \\
\text { OSA } \\
\text { nonobese }\end{array}$ & $\begin{array}{l}\geqslant 20 \\
\geqslant 20\end{array}$ & $\begin{array}{l}12 \\
14\end{array}$ & $\begin{array}{l}47 \pm 9.6^{\#} \\
50 \pm 9.8^{\#}\end{array}$ & $\begin{array}{l}34.9 \pm 3.4^{\#} \\
25.9 \pm 2.0^{\#}\end{array}$ & $\begin{array}{l}11 \pm 4.9^{\#} \\
13 \pm 4.8^{\#}\end{array}$ & $\begin{array}{l}\text { Baseline to } \\
\text { post-treatment }\end{array}$ & $\begin{array}{l}3 \text { months; } \\
12 \text { months }\end{array}$ & $5.7 \pm 1.4$ & $\begin{array}{l}\text { Leptin, } \\
\text { a.m., } \\
\text { fasted }\end{array}$ & $\begin{array}{l}\downarrow \text { leptin in } \\
\text { nonobese }\end{array}$ \\
\hline CHIN [32] & 1999 & OSA & $>20$ & 21 & $52.2 \pm 3.3$ & $29.3 \pm 1.1$ & $N / R$ & $\begin{array}{l}\text { Baseline to } \\
\text { post-treatment }\end{array}$ & $\begin{array}{l}3-4 \text { days; } \\
1 \text { month; } \\
6 \text { months }\end{array}$ & $N / R$ & $\begin{array}{l}\text { Leptin, } \\
\text { fasted }\end{array}$ & $\begin{array}{l}\downarrow \text { leptin after } \\
3-4 \text { days, } 1 \text { month } \\
\text { and } 6 \text { months }\end{array}$ \\
\hline CHIN [50] & 2003 & OSA & $N / R$ & 23 & $47 \pm 12.0^{\pi}$ & $31.0 \pm 4.0$ & $N / R$ & $\begin{array}{l}\text { Baseline to } \\
\text { post-treatment }\end{array}$ & $\begin{array}{c}1 \text { day } \\
(\mathrm{n}=23) ; \\
1 \text { month } \\
(\mathrm{n}=8)\end{array}$ & $4.3 \pm 1.4$ & $\begin{array}{l}\text { Leptin, } \\
\text { a.m., } \\
\text { fasted }\end{array}$ & $\begin{array}{l}\downarrow \text { leptin after } \\
1 \text { day and } \\
1 \text { month }\end{array}$ \\
\hline \multirow[t]{2}{*}{ CHIHARA [17] } & 2015 & OSA & $\geqslant 20$ & 21 & $54.6 \pm 12.4^{\#}$ & $26.5 \pm 3.9^{\#}$ & $11.1 \pm 5.5^{\#}$ & \multirow{2}{*}{$\begin{array}{l}\text { Baseline to } \\
\text { post-treatment and } \\
\text { between groups }\end{array}$} & \multirow[t]{2}{*}{3 months } & \multirow[t]{2}{*}{$4.5 \pm 2.0$} & \multirow[t]{2}{*}{$\begin{array}{l}\text { Leptin, } \\
\text { a.m., } \\
\text { fasted, p.p. }\end{array}$} & \multirow[t]{2}{*}{$\leftrightarrow$ leptin } \\
\hline & & Control & $<15$ & 15 & $54.3 \pm 14.3$ & $26.2 \pm 3.0^{\#}$ & $10.4 \pm 5.3^{\#}$ & & & & & \\
\hline Cuhadaroglu [33] & 2009 & $\begin{array}{l}\text { OSA-CPAP } \\
\text { compliant }\end{array}$ & $\geqslant 15$ & 31 & $53.9 \pm 9.7^{+}$ & $32.3 \pm 4.7$ & $11.3 \pm 5.7$ & $\begin{array}{l}\text { Baseline to } \\
\text { post-treatment }\end{array}$ & 2 months & $>4.0$ in all & $\begin{array}{l}\text { Leptin, } \\
\text { a.m., } \\
\text { fasted }\end{array}$ & $\downarrow$ leptin \\
\hline DRUMmond [57] & 2008 & OSA & $>20$ & 98 & $53.3 \pm 10.7$ & $33.2 \pm 5.0$ & $N / R$ & $\begin{array}{l}\text { Baseline to } \\
\text { post-treatment }\end{array}$ & $\begin{array}{l}1 \text { week; } \\
6 \text { months }\end{array}$ & $5.8 \pm 1.6$ & $\begin{array}{l}\text { Leptin, } \\
\text { a.m., } \\
\text { fasted }\end{array}$ & $\leftrightarrow$ leptin \\
\hline GARCIA [58] & 2011 & OSA & $\geqslant 15$ & 20 & $59.7 \pm 8.9$ & $36.5 \pm 8.0$ & $14.6 \pm 4.5$ & $\begin{array}{l}\text { Baseline to } \\
\text { post-treatment }\end{array}$ & 6 months & $5.3 \pm 0.4$ & $\begin{array}{l}\text { Leptin, } \\
\text { a.m., } \\
\text { fasted }\end{array}$ & $\leftrightarrow$ leptin \\
\hline $\mathrm{H}_{\text {ARSCH }}$ [9] & 2003 & $\begin{array}{l}\text { OSA } \\
\text { Control }\end{array}$ & $\begin{array}{l}\geqslant 30 \\
<5\end{array}$ & $\begin{array}{l}30 \\
30\end{array}$ & $\begin{array}{c}52 \pm 11.0 \\
51 \pm 5.5\end{array}$ & $\begin{array}{l}32.6 \pm 5.5 \\
30.6 \pm 3.3\end{array}$ & $\begin{array}{l}11 \pm 2.2 \\
5.1 \pm 1.6\end{array}$ & $\begin{array}{l}\text { Baseline to } \\
\text { post-treatment and } \\
\text { between-group }\end{array}$ & $\begin{array}{c}2 \text { days; } \\
2 \text { months }\end{array}$ & $>3.0$ & $\begin{array}{l}\text { Leptin, } \\
\text { a.m., } \\
\text { fasted }\end{array}$ & $\begin{aligned} & \downarrow \text { leptin after } \\
& 2 \text { months; } \\
\leftrightarrow & \text { control versus } \\
& \text { treatment }\end{aligned}$ \\
\hline $\mathrm{H}_{\text {ARSCH }}[51]$ & 2004 & OSA & $\bar{x}=46$ & 30 & $56.4 \pm 11.1$ & $32.3 \pm 6.5$ & $12.9 \pm 3.6$ & $\begin{array}{l}\text { Baseline to } \\
\text { post-treatment }\end{array}$ & $\begin{array}{c}2 \text { days; } \\
3 \text { months }\end{array}$ & $5.2 \pm 0.9$ & $\begin{array}{l}\text { Leptin, } \\
\text { a.m., } \\
\text { fasted }\end{array}$ & $\begin{array}{c}\leftrightarrow \text { leptin at } \\
2 \text { days; } \\
\downarrow \text { leptin trend } \\
\text { ( } p=0.058 \text { ) after } \\
3 \text { months }\end{array}$ \\
\hline $\mathrm{H}_{\mathrm{ARSCH}}[54]$ & 2004 & OSA & $\bar{x}=43$ & 40 & $53.8 \pm 11.8$ & $32.8 \pm 6.9$ & $12.9 \pm 3.6$ & $\begin{array}{c}\text { Baseline to } \\
\text { post-treatment }\end{array}$ & $\begin{array}{c}2 \text { days; } \\
3 \text { months }\end{array}$ & $5.2 \pm 0.9$ & $\begin{array}{l}\text { Leptin, } \\
\text { p.m., } \\
\text { fasted }\end{array}$ & $\begin{array}{l}\leftrightarrow \text { leptin at } \\
2 \text { days; } \\
\downarrow \text { leptin at } \\
3 \text { months }\end{array}$ \\
\hline
\end{tabular}




\begin{tabular}{|c|c|c|c|c|c|c|c|c|c|c|c|c|}
\hline First author [ref.] & Year & Participants & $\begin{array}{l}\text { AHI cut-off } \\
\text { events } \cdot h^{-1}\end{array}$ & $\begin{array}{l}\text { Sample } \\
\text { size }\end{array}$ & $\begin{array}{c}\text { Age } \\
\text { years } M / F\end{array}$ & $\begin{array}{c}\text { Baseline BMI } \\
\mathrm{kg} \cdot \mathrm{m}^{-2} \mathrm{M} / \mathrm{F}\end{array}$ & $\begin{array}{l}\text { Baseline } \\
\text { ESS M/F }\end{array}$ & Design & $\begin{array}{l}\text { CPAP } \\
\text { duration }\end{array}$ & $\begin{array}{c}\text { CPAP } \\
\text { compliance } h \text { per } \\
\text { night } M / F\end{array}$ & Outcomes & $\begin{array}{l}\text { Main findings: } \\
\text { effect of CPAP }\end{array}$ \\
\hline Hoyos [61] & 2012 & $\begin{array}{l}\text { Active CPAP } \\
\text { Sham CPAP }\end{array}$ & $\begin{array}{l}\geqslant 20 \\
\geqslant 20\end{array}$ & $\begin{array}{l}34 \\
31\end{array}$ & $\begin{array}{l}51.0 \pm 12.3 \\
46.4 \pm 10.4\end{array}$ & $\begin{array}{c}31.6 \pm 5.3 \\
31 \pm 5.1\end{array}$ & $\begin{array}{l}10.0 \pm 4.0 \\
10.2 \pm 4.8\end{array}$ & $\begin{array}{l}\text { Sham-controlled, } \\
\text { parallel; RCT }\end{array}$ & $\begin{array}{l}3 \text { months } \\
3 \text { months }\end{array}$ & $\begin{array}{l}N / R \\
N / R\end{array}$ & $\begin{array}{l}\text { Leptin, } \\
\text { a.m., } \\
\text { fasted }\end{array}$ & $\begin{array}{c}\leftrightarrow \text { leptin after } \\
\text { treatment } \\
\leftrightarrow \text { leptin between } \\
\quad \text { groups }\end{array}$ \\
\hline IP [10] & 2000 & OSA & $\geqslant 5$ & 9 & $43.6 \pm 10.1^{\#}$ & $27.0 \pm 2.9^{\#}$ & $N / R$ & $\begin{array}{l}\text { Baseline to } \\
\text { post-treatment }\end{array}$ & 6 months & $N / R$ & $\begin{array}{l}\text { Leptin, } \\
\text { a.m., } \\
\text { fasted }\end{array}$ & $\begin{array}{l}\downarrow \text { leptin at } \\
6 \text { months }\end{array}$ \\
\hline KRITIKOU [60] & 2014 & OSA & $\bar{x}=38$ & 35 & $\begin{array}{c}\text { Range } \\
41.7-66.3\end{array}$ & $28.6 \pm 0.6$ & $10.4 \pm 0.9$ & $\begin{array}{l}\text { Sham-controlled, } \\
\text { crossover; RCT }\end{array}$ & 2 months & $\begin{array}{l}\text { Active: } \\
6.1 \pm 1.2 \\
\text { Sham: } \\
5.3 \pm 1.2\end{array}$ & $\begin{array}{l}\text { Leptin, } \\
\text { a.m., } \\
\text { fasted }\end{array}$ & $\begin{array}{c}\leftrightarrow \text { leptin at } \\
2 \text { months or } \\
\text { between groups }\end{array}$ \\
\hline MURRI [59] & 2009 & OSA & $\geqslant 10$ & 78 & $52.3 \pm 11.3$ & $32.2 \pm 5.2$ & $15.3 \pm 5.5$ & $\begin{array}{l}\text { Baseline to } \\
\text { post-treatment }\end{array}$ & 1 month & $N / R$ & $\begin{array}{l}\text { Leptin, } \\
\text { a.m., } \\
\text { fasted }\end{array}$ & $\begin{array}{l}\leftrightarrow \text { leptin at } \\
1 \text { month }\end{array}$ \\
\hline SAARELAINEN [55] & 1997 & OSA & $N / R$ & 7 & $53.6 \pm 6.2$ & $34.4 \pm 4.9$ & $N / R$ & $\begin{array}{c}\text { Baseline to } \\
\text { post-treatment }\end{array}$ & 3 months & 5.6 & $\begin{array}{l}\text { Leptin, } \\
\text { a.m., } \\
\text { fasted }\end{array}$ & $\begin{array}{l}\downarrow \text { leptin at } \\
3 \text { months }\end{array}$ \\
\hline SANNER [52] & 2004 & OSA & $\geqslant 5$ & 68 & $57.5 \pm 11.0^{\S}$ & $31.2 \pm 5.6^{\S}$ & $N / R$ & $\begin{array}{l}\text { Baseline to } \\
\text { post-treatment }\end{array}$ & 6 months & $5.3 \pm 1.4$ & $\begin{array}{l}\text { Leptin, } \\
\text { a.m., } \\
\text { fasted }\end{array}$ & $\begin{array}{c}\downarrow \text { leptin at } \\
6 \text { months with } \\
\text { effective } \\
\text { treatment (AHI } \\
<5 \text { ) }\end{array}$ \\
\hline Shimizu [53] & 2002 & OSA & $\geqslant 20$ & 21 & $45.0 \pm 11.0$ & $28.9 \pm 3.7$ & $N / R$ & $\begin{array}{l}\text { Baseline to } \\
\text { post-treatment }\end{array}$ & 1 day & $N / R$ & $\begin{array}{l}\text { Leptin, } \\
\text { p.m., } \\
\text { unfasted, } \\
\text { overnight }\end{array}$ & $\begin{array}{c}\downarrow \text { leptin at } 03: 00 \mathrm{~h} \\
\text { and 06:30 h after } \\
\text { treatment }\end{array}$ \\
\hline TACHIKAWA [48] & 2016 & OSA & $>20$ & 63 & $60.6 \pm 10.0$ & $27.9 \pm 3.8$ & $8.7 \pm 5.3$ & $\begin{array}{l}\text { Baseline to } \\
\text { post-treatment }\end{array}$ & $\begin{array}{c}1 \text { day; } \\
3 \text { months }\end{array}$ & $4.5 \pm 1.6$ & $\begin{array}{l}\text { Leptin, } \\
\text { a.m., } \\
\text { fasted }\end{array}$ & $\begin{array}{c}\leftrightarrow \text { leptin after } \\
\text { treatment }\end{array}$ \\
\hline TAKAHASHI [18] & 2008 & OSA & $\bar{x}=46^{\pi}$ & 14 & $53.2 \pm 8.8$ & $28.5 \pm 3.7$ & $N / R$ & $\begin{array}{l}\text { Baseline to } \\
\text { post-treatment }\end{array}$ & $\begin{array}{l}3-4 \text { days; } \\
1 \text { month }\end{array}$ & $N / R$ & $\begin{array}{l}\text { Leptin, } \\
\text { a.m., } \\
\text { fasted }\end{array}$ & $\begin{array}{c}\leftrightarrow \text { leptin after } \\
\text { treatment }\end{array}$ \\
\hline TRENELL [56] & 2007 & $\begin{array}{l}\text { Regular } \\
\text { CPAP } \\
\text { Irregular } \\
\text { CPAP }\end{array}$ & $\begin{array}{l}>30^{f} \\
>30^{f}\end{array}$ & 19 & $49.0 \pm 12.0$ & $36.0 \pm 8.0$ & $\begin{array}{l}11.0 \pm 5.0 \\
12.0 \pm 6.0\end{array}$ & $\begin{array}{l}\text { Baseline to } \\
\text { post-treatment }\end{array}$ & 3 months & $\begin{array}{l}6.0 \pm 1.0 \\
2.0 \pm 2.0\end{array}$ & $\begin{array}{l}\text { Leptin, } \\
\text { a.m., } \\
\text { fasted }\end{array}$ & $\begin{array}{c}\downarrow \text { leptin in regular } \\
\text { users } \\
\leftrightarrow \text { leptin in } \\
\text { irregular users }\end{array}$ \\
\hline
\end{tabular}




\begin{tabular}{|c|c|c|c|c|c|c|c|c|c|c|c|c|}
\hline First author [ref.] & Year & Participants & $\begin{array}{l}\text { AHI cut-off } \\
\text { events } \cdot h^{-1}\end{array}$ & $\begin{array}{l}\text { Sample } \\
\text { size }\end{array}$ & $\begin{array}{c}\text { Age } \\
\text { years } M / F\end{array}$ & $\begin{array}{l}\text { Baseline BMI } \\
\mathrm{kg} \cdot \mathrm{m}^{-2} \mathrm{M} / \mathrm{F}\end{array}$ & $\begin{array}{l}\text { Baseline } \\
\text { ESS M/F }\end{array}$ & Design & $\begin{array}{l}\text { CPAP } \\
\text { duration }\end{array}$ & $\begin{array}{c}\text { CPAP } \\
\text { compliance } h \text { per } \\
\text { night } M / F\end{array}$ & Outcomes & $\begin{array}{l}\text { Main findings: } \\
\text { effect of CPAP }\end{array}$ \\
\hline \multicolumn{13}{|l|}{ Ghrelin } \\
\hline ChIHARA [17] & 2015 & $\begin{array}{l}\text { OSA } \\
\text { Control }\end{array}$ & $\begin{array}{l}\geqslant 20 \\
<15\end{array}$ & $\begin{array}{l}21 \\
15\end{array}$ & $\begin{array}{c}54.6 \pm 12.4^{\#} \\
54.3 \pm 14.3\end{array}$ & $\begin{array}{l}26.5 \pm 3.9^{\#} \\
26.2 \pm 3.0^{\#}\end{array}$ & $\begin{array}{l}11.1 \pm 5.5^{\#} \\
10.4 \pm 5.3^{\#}\end{array}$ & $\begin{array}{c}\text { Baseline to } \\
\text { post-treatment and } \\
\text { between groups }\end{array}$ & 3 months & $4.5 \pm 2.0$ & $\begin{array}{c}\text { Ghrelin, } \\
\text { a.m., } \\
\text { fasted, p.p. }\end{array}$ & $\begin{array}{l}\downarrow \text { fasted, p.p. } \\
\text { after treatment; } \\
\leftrightarrow \text { fasted p.p. in } \\
\text { OSA versus } \\
\text { control }\end{array}$ \\
\hline GARCIA $[58]$ & 2011 & OSA & $\geqslant 15$ & 20 & $59.7 \pm 8.9$ & $36.5 \pm 8.0$ & $14.6 \pm 4.5$ & $\begin{array}{l}\text { Baseline to } \\
\text { post-treatment }\end{array}$ & 6 months & $5.3 \pm 0.4$ & $\begin{array}{c}\text { Ghrelin, } \\
\text { a.m., } \\
\text { fasted, p.p. }\end{array}$ & $\begin{array}{l}\downarrow \text { fasted ghrelin } \\
\leftrightarrow \text { p.p. ghrelin }\end{array}$ \\
\hline $\mathrm{H}_{\text {ARSCH }}[9]$ & 2003 & $\begin{array}{l}\text { OSA } \\
\text { Control }\end{array}$ & $\begin{array}{l}\geqslant 30 \\
<5\end{array}$ & $\begin{array}{l}9 \\
9\end{array}$ & $\begin{array}{l}54 \pm 6.0 \\
49 \pm 6.0\end{array}$ & $\begin{array}{l}33.0 \pm 4.2 \\
33.9 \pm 3.9\end{array}$ & $\begin{array}{c}12.2 \pm 2.1 \\
5.1 \pm 1.6\end{array}$ & $\begin{array}{l}\text { Baseline to } \\
\text { post-treatment and } \\
\text { between groups }\end{array}$ & 2 days & $>3.0$ & $\begin{array}{l}\text { Ghrelin, } \\
\text { a.m., } \\
\text { fasted }\end{array}$ & $\begin{array}{l}\downarrow \text { ghrelin after } \\
2 \text { days; } \\
\leftrightarrow \text { control versus } \\
\quad \text { treatment }\end{array}$ \\
\hline TACHIKAWA [48] & 2016 & OSA & $>20$ & 63 & $60.6 \pm 10.0$ & $27.9 \pm 3.8$ & $8.7 \pm 5.3$ & $\begin{array}{c}\text { Baseline to } \\
\text { post-treatment }\end{array}$ & $\begin{array}{c}1 \text { day; } \\
3 \text { months }\end{array}$ & $4.5 \pm 1.6$ & $\begin{array}{l}\text { Ghrelin, } \\
\text { a.m., } \\
\text { fasted }\end{array}$ & $\begin{array}{c}\leftrightarrow \text { ghrelin after } \\
\text { treatment }\end{array}$ \\
\hline TAKAHASHI [18] & 2008 & OSA & $\bar{x}=46$ & 21 & $52.5 \pm 8.7$ & $28.8 \pm 3.8$ & $N / R$ & $\begin{array}{l}\text { Baseline to } \\
\text { post-treatment }\end{array}$ & $\begin{array}{l}3-4 \text { days; } \\
1 \text { month }\end{array}$ & $5.5 \pm 1.3$ & $\begin{array}{l}\text { Ghrelin, } \\
\text { a.m., } \\
\text { fasted }\end{array}$ & $\begin{array}{c}\leftrightarrow \text { after } 3-4 \text { days; } \\
\downarrow \text { ghrelin after } \\
1 \text { month }\end{array}$ \\
\hline \multicolumn{13}{|l|}{ Orexin } \\
\hline Busquets [62] & 2004 & $\begin{array}{c}\text { OSA } \\
\text { Control } \\
\text { OSA-CPAP }\end{array}$ & $\begin{array}{l}\bar{x}=54 \\
N / R \\
\bar{x}=8\end{array}$ & $\begin{array}{l}27 \\
13 \\
14\end{array}$ & $\begin{array}{c}52 \pm 10.4 \\
46 \pm 7.2 \\
57 \pm 11.2\end{array}$ & $\begin{array}{l}31 \pm 5.2 \\
24 \pm 3.6 \\
36 \pm 3.7\end{array}$ & $\begin{array}{c}10 \pm 15.2 \\
3 \pm 3.6 \\
5 \pm 3.7\end{array}$ & Between groups & $\geqslant 1$ year & $4.5 \pm 0.5$ & $\begin{array}{l}\text { Orexin, } \\
\text { 12:00 h }\end{array}$ & $\begin{array}{l}\downarrow \text { orexin in CPAP } \\
\text { versus control }\end{array}$ \\
\hline IGARASHI [63] & 2003 & OSA & $>20$ & 12 & $45.3 \pm 13.7^{\#}$ & $28.6 \pm 4.9^{\#}$ & $13.2 \pm 3.8^{\#}$ & $\begin{array}{l}\text { Baseline to } \\
\text { post-treatment }\end{array}$ & $3-4$ months & $N / R$ & $\begin{array}{l}\text { Orexin, } \\
\text { a.m., } \\
\text { fasted }\end{array}$ & $\begin{array}{c}\downarrow \text { orexin after } \\
\text { treatment }\end{array}$ \\
\hline SAKURAI [64] & 2005 & $\begin{array}{l}\text { OSA, AI } \geqslant 60 \\
\text { OSA, AI }<60\end{array}$ & $\begin{array}{l}\geqslant 20 \\
\geqslant 20\end{array}$ & $\begin{array}{l}11 \\
16\end{array}$ & $\begin{array}{l}50.2 \pm 17.2 \\
57.5 \pm 10.4\end{array}$ & $\begin{array}{l}28.9 \pm 5.6 \\
26.0 \pm 2.8\end{array}$ & $\begin{array}{l}17.1 \pm 2.6 \\
10.8 \pm 2.8\end{array}$ & $\begin{array}{l}\text { Baseline to } \\
\text { post-treatment }\end{array}$ & $3-6$ months & $>4.0$ in all & $\begin{array}{l}\text { Orexin, } \\
\text { a.m., } \\
\text { fasted }\end{array}$ & $\begin{array}{c}\uparrow \text { orexin after } \\
\text { treatment } \\
\leftrightarrow \text { orexin after } \\
\text { treatment }\end{array}$ \\
\hline \multicolumn{13}{|l|}{ NPY } \\
\hline BARCELo [7] & 2005 & $\begin{array}{l}\text { OSA obese } \\
\text { OSA } \\
\text { nonobese }\end{array}$ & $\begin{array}{l}\geqslant 20 \\
\geqslant 20\end{array}$ & $\begin{array}{l}12 \\
14\end{array}$ & $\begin{array}{l}47 \pm 9.6^{\#} \\
50 \pm 9.8^{\#}\end{array}$ & $\begin{array}{l}34.9 \pm 3.4^{\#} \\
25.9 \pm 2.0^{\#}\end{array}$ & $\begin{array}{l}11 \pm 4.9^{\#} \\
13 \pm 4.8^{\#}\end{array}$ & $\begin{array}{c}\text { Baseline to } \\
\text { post-treatment }\end{array}$ & $\begin{array}{l}3 \text { months; } \\
12 \text { months }\end{array}$ & $5.7 \pm 1.4$ & $\begin{array}{l}\text { NPY, } \\
\text { a.m., } \\
\text { fasted }\end{array}$ & $\begin{array}{c}\downarrow N P Y \text { in obese } \\
\text { and nonobese at } \\
12 \text { months }\end{array}$ \\
\hline
\end{tabular}

Data are presented as $n$ or mean $\pm S D$, unless otherwise stated. AHI: apnoea-hypopnoea index; M: male; F: female; BMI: body mass index; ESS: Epworth sleepiness scale; NPY: neuropeptide Y; N/R: not reported; p.p.: post-prandial; RCT: randomised controlled trial; Al: arousal index. $\uparrow$ : significant increase; $\downarrow$ : significant decrease; $\leftrightarrow$ : no difference; $\#$ : values represent full OSA group at baseline, not just CPAP; ๆ: values represent full CPAP group, not just those with leptin sampling; ${ }^{+}$: values represent full group including compliant and noncompliant CPAP users; ${ }^{\S}$ : values represent full OSA treatment group, not just those treated with CPAP; ${ }^{f}$ : cut-off based on respiratory disturbance index not AHI. 
orexin when sampled in the morning in the fasted state [63]. In a comparison between controls, untreated OSA patients and OSA patients who had used CPAP for $\geqslant 1$ year, levels were significantly lower in both treated and untreated patients versus controls, suggesting CPAP is not associated with a normalisation of orexin levels [62]. However, because of the cross-sectional between-group nature of the study, it is difficult to conclude an effect of treatment. A third study stratified OSA patients based on having an arousal index of $\geqslant 60$ (group A) or $<60$ (group B) [64]. The authors reported that compared to baseline, orexin levels were significantly increased in group A but not group B after 3-6 months of treatment [64]. Participants in the study that demonstrated an increase in orexin in severe OSA after treatment all had good compliance ( $>4$ h per night) [64], whereas the study that demonstrated a decrease in orexin after treatment did not report on duration of nightly CPAP use [63].

The single study to date which investigated neuropeptide Y (NPY) (another hypothalamic neuropeptide that increases food intake) in response to CPAP compared values obtained in the morning under fasting conditions at baseline, and after 3- and 12-month follow-ups in obese and nonobese OSA patients [7]. NPY levels were found to decrease significantly after 12 months under CPAP in both obese and nonobese patients [7].

\section{Energy expenditure via indirect calorimetry}

Findings on the effects of CPAP on energy expenditure assessed using indirect calorimetry are summarised in table 3. The effects of CPAP on RMR were investigated in three studies using ventilated hood/canopy indirect calorimetry $[48,65,66]$, and findings are mixed. After a single night of CPAP, RMR values remained significantly higher than controls, indicating a lack of treatment effect in lowering RMR [65]. However, the between-group design and short duration of treatment in that study make a conclusion on the effect of treatment difficult. A comparison of baseline versus 2-month [67] and 3-month [66] follow-up found no effect of treatment on RMR, whereas a pre-post design observed a significant reduction in basal metabolic rate after 3 months of treatment, but not 1 day, compared to baseline in 63 patients with AHI $>20$ [48]. It should be noted that the baseline BMI of participants in the studies that did not observe changes in RMR $[66,67]$ were higher than in the study that did [48], possibly accounting for discrepancies (table 3).

When measured using whole-room indirect calorimetry (WRIC), RMR was found to be unchanged after 3 months CPAP versus baseline [26]. However, that same study did observe that compared to baseline, 3 months of CPAP resulted in a significant reduction in sleeping metabolic rate (SMR), despite unchanged 24-h energy expenditure [26]. Contrasting results were observed in a recent pilot study which also utilised WRIC to determine the effects of CPAP on energy expenditure [68]. In that crossover placebo-controlled trial (not randomised), 2 months of active CPAP resulted in significantly increased SMR and 24-h energy expenditure versus placebo CPAP [68]. The findings were interpreted to suggest that CPAP may correct for the OSA-related adaptive decreased in thermogenesis. However, the pilot study suffered from a small sample size $(\mathrm{n}=3)$ and a lack of randomisation (all patients received active followed by sham), so findings should be replicated.

\section{Energy expenditure via physical activity}

Findings on the effects of CPAP on physical activity-related energy expenditure are summarised in table 4. Three studies utilised subjective, questionnaire-based measures of physical activity [47, 69, 70], and 12 studies [34, 38, 48, 49,71-78], including three conference abstracts $[72,73,75]$, assessed physical activity using objective measures.

Subsequent to the APPLES baseline study mentioned above [79], participants matched for sex, BMI and ESS and with AHI $\geqslant 10$ events. $\mathrm{h}^{-1}$ were randomised to receive sham or active CPAP in an RCT, and physical activity was assessed at follow-up using the Arizona activity frequency questionnaire [47]. In the 4-month follow-up assessment ( $\mathrm{n}=114$ sham; $\mathrm{n}=117$ active), no difference was observed in total energy expenditure; however, recreational calories expended were significantly increased in women in the CPAP group at 4 months versus baseline [47]. Collecting physical activity data using the general practitioners physical activity questionnaire in CPAP-compliant patients ( $\geqslant 4 \mathrm{~h}$ per night for $\geqslant 70 \%$ of a 2 -week period) at 2 weeks, 3 months and 6 months post-baseline follow-up, significant increases in the number of daily hours of activity were observed during all follow-up time points versus baseline [69]. In an RCT comparing 3 months CPAP treatment with conservative treatment control, no between-group difference in physical activity quantified as minutes per week, based on the international physical activity questionnaire was found [70].

A double-blind, randomised, sham-controlled CPAP trial monitored physical activity levels via SenseWear (BodyMedia, Pittburgh, PA, USA) at baseline and after 4 weeks [71]. Both active and sham groups showed increased physical activity at follow-up, but the active group had more steps than the sham group, indicating increased physical activity after active versus sham CPAP. It should be noted that the active group 
TABLE 3 Summary of effects of continuous positive airway pressure (CPAP) on energy expenditure via indirect calorimetry in obstructive sleep apnoea (OSA) patients

\begin{tabular}{|c|c|c|c|c|c|c|c|c|c|c|c|c|}
\hline $\begin{array}{l}\text { First author } \\
\text { [ref.] }\end{array}$ & Year & Participants & $\begin{array}{l}\text { AHI cut-off } \\
\text { events } \cdot h^{-1}\end{array}$ & $\begin{array}{l}\text { Sample } \\
\text { size }\end{array}$ & Age years & $\begin{array}{l}\text { Baseline } \\
\text { BMI } \mathrm{kg} \cdot \mathrm{m}^{-2}\end{array}$ & $\begin{array}{l}\text { Baseline } \\
\text { ESS }\end{array}$ & Design & $\begin{array}{l}\text { CPAP } \\
\text { duration }\end{array}$ & $\begin{array}{c}\text { CPAP } \\
\text { compliance h } \\
\text { per night }\end{array}$ & Outcomes & $\begin{array}{l}\text { Main findings: } \\
\text { effect of CPAP }\end{array}$ \\
\hline FEKETE [65] & 2015 & $\begin{array}{l}\text { OSA-CPAP } \\
\text { Control }\end{array}$ & $\begin{array}{l}\geqslant 15^{\#} \\
N / R\end{array}$ & $\begin{array}{l}62 \\
19\end{array}$ & $\begin{array}{l}46.5 \pm 11.4 \\
50.8 \pm 11.7\end{array}$ & $\begin{array}{l}33.9 \pm 5.2 \\
28.3 \pm 3.1\end{array}$ & $\begin{array}{c}13.0 \pm 6.1 \\
8.5 \pm 3.4\end{array}$ & Between groups & 1 day & $N / R$ & $\begin{array}{l}\text { REE using } \\
\text { indirect } \\
\text { calorimetry, } \\
\text { pre-sleep and } \\
\text { after awakening }\end{array}$ & $\begin{array}{c}\uparrow \text { REE and REE/ } \\
\text { LBM in } \\
\text { OSA-CPAP } \\
\text { versus control }\end{array}$ \\
\hline Ryan [66] & 1995 & OSA & $>30$ & 10 & $45.0 \pm 10.0^{\pi}$ & $39.8 \pm 6.5^{\uparrow}$ & $N / R$ & $\begin{array}{l}\text { Baseline to } \\
\text { post-treatment }\end{array}$ & 3 months & $5.0 \pm 1.0$ & $\begin{array}{l}\text { REE using } \\
\text { indirect } \\
\text { calorimetry, a. } \\
\text { m., fasted; } \\
\text { TEF after } \\
\text { liquid test meal }\end{array}$ & $\begin{array}{c}\leftrightarrow \text { REE after } \\
\text { treatment } \\
\leftrightarrow \text { TEF after } \\
\text { treatment }\end{array}$ \\
\hline SHECHTER [68] & 2015 & OSA & $\bar{x}=28$ & 3 & $50.7 \pm 4.5$ & $32.1 \pm 1.4$ & $10.0 \pm 1.7$ & $\begin{array}{c}\text { Sham-controlled, } \\
\text { crossover }\end{array}$ & 2 months & $\mathrm{N} / \mathrm{R}$ & $\begin{array}{l}\text { WRIC for 24-h } \\
\text { energy } \\
\text { expenditure } \\
\text { and sleeping } \\
\text { energy } \\
\text { expenditure }\end{array}$ & $\begin{array}{l}\uparrow \text { mean daily } \\
\text { energy } \\
\text { expenditure and } \\
\text { sleeping energy } \\
\text { expenditure in } \\
\text { active versus } \\
\text { sham }\end{array}$ \\
\hline SMURRA [67] & 2001 & OSA & $\bar{x}=31$ & 10 & $49.2 \pm 8.7$ & $33.0 \pm 3.8$ & $N / R$ & $\begin{array}{l}\text { Baseline to } \\
\text { post-treatment }\end{array}$ & 2 months & $6.4 \pm 0.8$ & $\begin{array}{c}\text { Indirect } \\
\text { calorimetry for } \\
\text { basal energy } \\
\text { expenditure }\end{array}$ & $\begin{array}{c}\leftrightarrow \text { basal energy } \\
\text { expenditure } \\
\text { after treatment }\end{array}$ \\
\hline StenLof [26] & 1996 & OSA & $\geqslant 20^{+}$ & 5 & $46.0 \pm 13.0$ & $34.0 \pm 7.0$ & $\mathrm{~N} / \mathrm{R}$ & $\begin{array}{l}\text { Baseline to } \\
\text { post-treatment }\end{array}$ & 3 months & $N / R$ & $\begin{array}{l}\text { WRIC for } 24 \mathrm{~h} \\
\text { energy } \\
\text { expenditure; } \\
\text { BMR in a.m., } \\
\text { fasted; SMR }\end{array}$ & $\begin{array}{c}\downarrow \mathrm{SMR} / \mathrm{FFM} \\
\text { after treatment } \\
\leftrightarrow \text { BMR or } 24 \mathrm{~h} \\
\text { energy } \\
\text { expenditure }\end{array}$ \\
\hline TACHIKAWA [48] & 2016 & OSA & $>20$ & 63 & $60.6 \pm 10.0$ & $27.9 \pm 3.8$ & $8.7 \pm 5.3$ & $\begin{array}{l}\text { Baseline to } \\
\text { post-treatment }\end{array}$ & $\begin{array}{c}1 \text { day; } \\
3 \text { months }\end{array}$ & $4.5 \pm 1.6$ & $\begin{array}{c}\text { Indirect } \\
\text { calorimetry for } \\
\text { BMR in a.m., } \\
\text { fasted }\end{array}$ & $\begin{array}{c}\downarrow \text { BMR after } \\
3 \text { months but not } \\
1 \text { day }\end{array}$ \\
\hline
\end{tabular}

Data are presented as $n$ or mean $\pm S D$, unless otherwise stated. AHI: apnoea-hypopnoea index; BMI: body mass index; ESS: Epworth sleepiness scale; N/R: not reported; REE: resting energy expenditure; LBM: lean body mass; TEF: thermic effect of food; WRIC: whole-room indirect calorimetry; SMR: sleeping metabolic rate; FFM: fat-free mass; BMR: basal metabolic rate. $\uparrow$ : significant increase; $\downarrow$ : significant decrease; $\leftrightarrow$ : no difference. \#: included AHI $\geqslant 15$ with no symptoms or $\geqslant 5$ with symptoms (daytime sleepiness, poor sleep or snoring/gasping); rate. $\uparrow$ : significant increase; $\downarrow$ : significant decrease; $\leftrightarrow$ : no difference. ${ }^{*}$ : included AHI $\geqslant 15$ with no sym
${ }^{\text {I }}$ : full OSA group at baseline, not just CPAP. ${ }^{+}:$cut-off based on respiratory disturbance index, not AHI. 
had a significantly higher baseline AHI than the sham group (50.2 versus 26.5 events. $\mathrm{h}^{-1}$ ). Two studies were conducted by investigators utilising wrist-worn accelerometry to document physical activity levels in a randomised, sham-controlled CPAP trial of 3 months of treatment [77, 78]. No between-group difference was seen when focusing on the most active and least active periods of the day [78] or over the 24-h recording [77]. For both studies, mean CPAP use during treatment periods in both groups was low $(<4 \mathrm{~h}$ per night) [77, 78], which may account for a lack of effect. However, a separate study which included only compliant CPAP users ( $>5 \mathrm{~h}$ per night) and compared baseline to follow-up (range 6-11 months) reported no changes post-CPAP in physical activity or energy expenditure as derived from ankle-bound accelerometry [34]. Consistent with this, an RCT which ensured high compliance of 8-h nightly CPAP use for 2 weeks found no difference in wrist accelerometry-derived physical activity levels compared to oral placebo [49]. Two studies also failed to note changes in objective physical activity levels after 3 [48] or 4 months [76] of treatment (both RCTs), whereas a longer RCT of 6 months of treatment observed a decrease in sedentary time but no accompanying increase in moderate-to-vigorous physical activity levels or steps per day [38].

The findings of conference reports suggest a positive effect of CPAP, with trends for increased physical activity and daily energy expenditure [73], as well as increased nonexercise activity thermogenesis [75]. However, these preliminary findings, which are based on small sample sizes and are less fully described, should be expanded upon. Another conference abstract described a significantly increased number of pedometer-recorded steps per day in 62 participants with at least mild OSA after CPAP for 3 and 7 months compared to baseline [72].

Finally, a decrease in wrist-actigraphy derived physical activity during the night, indicative of reduced movement arousals during sleep, was reported in patients after 2 months of CPAP versus baseline [74]. These findings are in accordance with a reduced AHI, and may be functionally related to the aforementioned findings of reduced SMR [26].

\section{Discussion}

Summary and considerations

Food intake

Measuring food intake is critical when considering how CPAP affects energy balance. The effects of CPAP on energy intake have been under-studied, with only four published investigations to date [38, 47-49]. In general, CPAP has not been found to affect energy intake [47, 80], although increased energy intake was seen in individuals who gained weight after treatment [48]. A positive feature of the energy intake studies is that most were from RCTs. This strengthens the designs, although the small number of investigations suggests that more RCTs on this topic should be conducted. It should be noted that a major limitation in these investigations is that the monitoring of food intake was conducted with self-reported measures, such as the FFQ and food logs. Based on its subjective nature, the FFQ has been criticised as an inaccurate measure, characterised by a systematic underestimation of actual energy intake [81]. Similar under-reporting of actual food intake based on 2-week self-reported food records [82] and diet diary techniques [83] have been reported. It was suggested that these subjective tools may lead to inaccurate conclusions and, accordingly, should be abandoned [81]. A critical future step is to conduct systematic investigations of food choice and intake in response to CPAP using objective measures under both real-life and controlled laboratory conditions.

\section{Hormonal regulation of food intake}

Considerable work has been undertaken to investigate how CPAP affects hunger/satiety hormones. Findings of reduced leptin levels after CPAP, even without concurrent weight loss, were described by some [7, 9, 10, $32,33,50-56]$, but not all prospective observational CPAP studies [17, 18, 48, 57-59]. Importantly, the only two sham-controlled RCTs conducted to date investigating the effects of CPAP on leptin reported no effect of treatment $[60,61]$. Since leptin is secreted by adipose tissue, with concentrations increasing with adipose mass [84], an effect of CPAP on body fat content could also influence leptin levels. The RCT by Hoyos et al. [61], as well as the findings of a recent meta-analysis [85], indicates that CPAP does not reduce visceral fat, and therefore changes in body fat are unlikely to be influencing leptin secretion after CPAP. An alternative explanation is that leptin levels may decrease after CPAP use because of a reduced need to stimulate ventilatory drive [86]. Specifically, hypoxaemia enhances leptin expression [87], and it has been suggested that elevated leptin in OSA occurs as a consequence of hypoxia as opposed to fat accumulation [88]. Similar to leptin, most of the prospective studies have demonstrated that CPAP treatment for as little as 2 days [9] to as long as 6 months [58] is effective in reducing ghrelin versus baseline. Taken together, it appears that a potential decrease in the leptin-associated satiety signal, and therefore risk of increased food intake, is compensated for by the concomitant decrease in ghrelin levels after CPAP. Alternatively, an alleviation of leptin resistance (i.e. reduced efficacy of leptin's satiety-inducing effects due to a hypersecretion) together with a concomitant decrease in ghrelin secretion after CPAP may be occurring. 
The findings of an improvement in the hunger/satiety hormones are therefore encouraging from a clinical perspective.

In terms of methodology, more RCTs should be conducted, since only two leptin studies were RCTs, and no other investigations of hunger/satiety hormones. It is critical to control for BMI, as well as time of sampling and feeding state, as many of the hunger/satiety-regulating hormones either show a diurnal variation or are responsive to food intake. Leptin [89, 90], orexin [91] and possibly ghrelin [92] show diurnal/circadian rhythms. Leptin levels are stimulated by food intake [93], whereas ghrelin levels decrease after a meal [94], so investigators should control for this confounding factor. Although the data provide a potential mechanistic basis involving leptin, ghrelin, orexin and/or NPY for an improvement in food intake within OSA patients after CPAP, more studies are required to convincingly link the hormonal control to food intake patterns after CPAP.

\section{Energy expenditure via indirect calorimetry}

Like energy intake, it is essential to consider energy expenditure to understand how CPAP affects body weight regulation. Also like energy intake, the effects of CPAP on indirect calorimetry-derived measures of energy expenditure such as RMR and SMR have been under-studied, with only six trials conducted to date. Studies have varied in methodology (canopy versus whole-room), treatment duration (1 day versus 2 months versus 3 months), baseline BMI and comparison groups (pre-post treatment versus placebo-controlled). No RCTs on indirect calorimetry-derived energy expenditure have been conducted to date. In terms of methodology used to measure energy expenditure, it is important to note whether indirect calorimetry is done via canopy or WRIC. This is because RMR assessments via the canopy/hood system will mainly be determined by participant fat-free mass [95], whereas the WRIC assessments will additionally register energy expenditure from nonexercise activity thermogenesis and possibly exercise energy expenditure, depending on protocol [68]. Interestingly, whereas no changes in visceral fat were observed in the RCT by Hoyos et al. [61], CPAP was found to increase lean mass, which has the potential to favourably influence energy metabolism and exercise capacity.

In addition to observations of no change in response to treatment, CPAP may either correct for an abnormally high OSA-related energy expenditure by reducing sleeping [96] or resting energy expenditure, or alternatively, it may act to increase daily and sleeping energy expenditure [68] as a means of correcting for an OSA-related decrease in thermogenesis. While placebo-controlled trials are often viewed as the gold standard for determining intervention effects, the use of sham CPAP has been found to increase arousal index and reduce sleep efficiency [97]. It is therefore possible that this unintentional sleep disruption may affect sleeping energy expenditure, or possibly next-day RMR. This may also account for the discrepancies between findings of effects of CPAP on SMR, described earlier. Another important consideration is baseline BMI, as this parameter is known to influence energy metabolism. Finally, the majority of studies on indirect calorimetry-derived energy expenditure described here were performed primarily on male participants. Specifically, four of the six studies included $100 \%$ male OSA patients $[26,44,67,68]$, with the remaining studies composed of $92 \%$ [66] or $81 \%$ [48] male OSA patients. Accordingly, there may be important sex-based effects which are not apparent based on the current literature. Based on the limited number of studies and inconsistencies, more studies should be conducted before definitive conclusions can be made. It is still too early to conclude that CPAP use causes weight gain because of a reduction in energy expenditure, as has been suggested $[35,48]$.

The regulation of energy expenditure is multifactorial, as total energy expenditure is the summation of several components, including RMR, the thermic effect of food (i.e. energy associated with absorption and metabolism of food) and physical activity [98]. Therefore, full 24-h assessments of energy expenditure should be performed in response to CPAP under controlled conditions, as was done in two investigations $[26,68]$. Another option to measure total energy expenditure after CPAP is with the use of doubly labelled water [99, 100], which is suited for measures of long-term free-living energy expenditure. Such an approach may be favourable for investigators who do not have access to WRIC facilities. Furthermore, it was observed that free-living energy expenditure estimated with doubly labelled water is $15 \%$ greater than total energy expenditure measured via WRIC [101]. This potential underestimation of energy expenditure from the latter method is likely due to the confines of the small metabolic chambers, in which participants' physical activity levels are relatively restricted.

\section{Energy expenditure via physical activity}

A large and important component of total energy expenditure is physical activity. Physical activity levels in response to CPAP were assessed either subjectively via questionnaire $[47,69,70]$ or objectively via accelerometer [34, 38, 48, 49, 71-78]. Importantly, of the 12 studies that objectively monitored physical activity levels, half were RCTs. Whereas objective monitoring is preferable, the site of attachment of 
TABLE 4 Summary of effects of continuous positive airway pressure (CPAP) on physical activity levels in obstructive sleep apnoea (OSA) patients

\begin{tabular}{|c|c|c|c|c|c|c|c|c|c|c|c|c|}
\hline Author [ref.] & Year & Participants & $\begin{array}{l}\text { AHI cutoff } \\
\text { events } h^{-1}\end{array}$ & $\begin{array}{l}\text { Sample } \\
\text { size }\end{array}$ & $\begin{array}{c}\text { Age } \\
\text { years } M / F\end{array}$ & $\begin{array}{l}\text { Baseline } \\
\text { BMI } \mathrm{kg} \cdot \mathrm{m}^{-2}\end{array}$ & $\begin{array}{l}\text { Baseline } \\
\text { ESS }\end{array}$ & Design & $\begin{array}{l}\text { CPAP } \\
\text { duration }\end{array}$ & $\begin{array}{c}\text { CPAP } \\
\text { compliance } h \text { per } \\
\text { night } M / F\end{array}$ & Outcomes & $\begin{array}{l}\text { Main findings: } \\
\text { effect of CPAP }\end{array}$ \\
\hline \multicolumn{13}{|l|}{$\begin{array}{l}\text { Questiornnaire-based } \\
\text { assessment }\end{array}$} \\
\hline \multirow[t]{2}{*}{ Batool-Anwar [47] } & 2014 & Active CPAP & $\geqslant 10$ & 117 & $\begin{array}{l}54.0 \pm 13.0 / \\
55.0 \pm 13.0\end{array}$ & $\begin{array}{c}33.0 \pm 6.3^{*} / 36.0 \\
\pm 11.0\end{array}$ & $\begin{array}{c}10.0 \pm 4.0 / \\
10.7 \pm 4.6\end{array}$ & $\begin{array}{l}\text { Sham-controlled, } \\
\text { parallel; RCT }\end{array}$ & 4 months & $\begin{array}{r}4.0 \pm 2.9 * * \\
/ 3.5 \pm 2.8\end{array}$ & $\begin{array}{l}\text { Physical activity } \\
\text { questionnaire }\end{array}$ & $\begin{array}{l}\leftrightarrow \text { total energy } \\
\text { expenditure; } \\
\uparrow \text { recreational }\end{array}$ \\
\hline & & Sham CPAP & $\geqslant 10$ & 114 & $\begin{array}{l}55.0 \pm 14.0 / \\
54.0 \pm 13.0\end{array}$ & $\begin{array}{c}31.0 \pm 5.5^{*} / \\
34.0 \pm 8.8\end{array}$ & $\begin{array}{l}9.7 \pm 4.3 / \\
9.7 \pm 3.7\end{array}$ & & & 4 months & $2.6 \pm 2.6 * * / 2.9 \pm 2.6$ & $\begin{array}{l}\text { calories expended in } \\
\text { females in CPAP } \\
\text { group at } 4 \text { months }\end{array}$ \\
\hline BILLINGS [69] & 2013 & $\begin{array}{l}\text { OSA-CPAP } \\
\text { compliant }\end{array}$ & $\geqslant 15$ & 21 & $50.5 \pm 2.0$ & $36.0 \pm 2.0$ & $15.4 \pm 0.7$ & $\begin{array}{l}\text { Baseline to } \\
\text { post-treatment }\end{array}$ & $\begin{array}{c}2 \text { weeks; } 3 \\
\text { months; } 6 \\
\text { months }\end{array}$ & $\begin{array}{c}2 \text { weeks } 5.2 \pm 0.3 \\
3 \text { months } 5.1 \pm 0.3 \\
6 \text { months } 5.7 \pm 0.3\end{array}$ & $\begin{array}{l}\text { Physical activity } \\
\text { questionnaire }\end{array}$ & $\begin{array}{c}\uparrow \text { daily } \\
\text { hours of activity; } \\
\uparrow \text { strenuous weekly } \\
\text { activity }\end{array}$ \\
\hline \multirow[t]{2}{*}{ SALORD [70] } & 2015 & CPAP & $>30$ & 42 & $48.5 \pm 8.6$ & $45.7 \pm 5.0$ & $7.9 \pm 4.5$ & $\begin{array}{l}\text { Placebo-controlled, } \\
\text { parallel; RCT }\end{array}$ & 3 months & $5.4 \pm 1.6$ & $\begin{array}{l}\text { Physical activity } \\
\text { questionnaire }\end{array}$ & $\begin{array}{c}\leftrightarrow \text { METS min per } \\
\text { week between groups }\end{array}$ \\
\hline & & CT & $>30$ & 38 & $44.6 \pm 9.4$ & $49.3 \pm 6.6^{*}$ & $7.9 \pm 5.2$ & & 3 months & & & \\
\hline \multicolumn{13}{|l|}{$\begin{array}{l}\text { Accelerometry-based } \\
\text { assessment }\end{array}$} \\
\hline Chasens [71] & 2014 & $\begin{array}{l}\text { Active CPAP } \\
\text { Sham CPAP }\end{array}$ & $\begin{array}{l}\geqslant 10 \\
\geqslant 10\end{array}$ & $\begin{array}{l}23 \\
12\end{array}$ & $55.6 \pm 10.6$ & $35.5 \pm 6.2$ & $\begin{array}{l}11.4 \pm 4.6 \\
10.6 \pm 3.7\end{array}$ & $\begin{array}{l}\text { Placebo-controlled, } \\
\text { parallel; RCT }\end{array}$ & $\begin{array}{l}4 \text { weeks } \\
8 \text { weeks }\end{array}$ & $\begin{array}{c}96-489 \text { min per } \\
\text { night }\end{array}$ & $\begin{array}{l}\text { SenseWear } \\
\text { armband }\end{array}$ & $\begin{array}{c}\uparrow \text { steps walked in } \\
\text { active versus sham } \\
\text { CPAP }\end{array}$ \\
\hline DiamantI [34] & 2013 & $\begin{array}{l}\text { OSA-CPAP } \\
\text { compliant }\end{array}$ & $\geqslant 15$ & 24 & $51.9 \pm 10.6$ & $34.4 \pm 6.5$ & $8.3 \pm 4.0$ & $\begin{array}{l}\text { Baseline to } \\
\text { post-treatment }\end{array}$ & $\begin{array}{c}\text { 6-11 } \\
\text { months }\end{array}$ & $6.3 \pm 0.8$ & $\begin{array}{c}\text { Ankle } \\
\text { accelerometer }\end{array}$ & $\begin{array}{c}\leftrightarrow \text { energy expenditure } \\
\text { or steps per day after } \\
\text { treatment }\end{array}$ \\
\hline IGELSTROM [38] & 2014 & OSA & $\geqslant 15$ & 35 & $53.0 \pm 11.0$ & $33.6 \pm 4.3$ & $N / R$ & $\begin{array}{c}\text { Baseline to } \\
\text { post-treatment; RCT }\end{array}$ & 6 months & $\begin{array}{l}>4 \text { in } 73 \% \text { of } \\
\text { participants }\end{array}$ & $\begin{array}{l}\text { SenseWear } \\
\text { armband }\end{array}$ & $\begin{array}{c}\downarrow \text { sedentary time after } \\
\text { treatment } \\
\leftrightarrow \text { MVPA or steps per } \\
\text { day }\end{array}$ \\
\hline JEAN [72] & 2015 & OSA & $>5$ & 62 & $53.0 \pm 13.0$ & $38.0 \pm 11.0$ & $N / R$ & $\begin{array}{l}\text { Baseline to } \\
\text { post-treatment }\end{array}$ & $\begin{array}{l}3 \text { months } \\
7 \text { months }\end{array}$ & $\begin{array}{l}>4 \text { in } 73 \% \text { of } \\
\text { participants }\end{array}$ & Pedometer & $\begin{array}{l}\uparrow \text { steps per day at } 3 \\
\text { and } 7 \text { month } \\
\text { follow-up }\end{array}$ \\
\hline KERLEY [73] & 2012 & OSA & $N / R$ & 15 & 54.7 & $N / R$ & $N / R$ & $\begin{array}{l}\text { Baseline to } \\
\text { post-treatment }\end{array}$ & 3 months & $N / R$ & $\begin{array}{l}\text { SenseWear } \\
\text { armband }\end{array}$ & $\begin{array}{c}\text { Trend toward } \uparrow \text { total } \\
\text { daily energy } \\
\text { expenditure and } \\
\text { physical activity }\end{array}$ \\
\hline
\end{tabular}




\begin{tabular}{|c|c|c|c|c|c|c|c|c|c|c|c|c|}
\hline Author [ref.] & Year & Participants & $\begin{array}{l}\text { AHI cutoff } \\
\text { events } \cdot h^{-1}\end{array}$ & $\begin{array}{l}\text { Sample } \\
\text { size }\end{array}$ & $\begin{array}{c}\text { Age } \\
\text { years M/F }\end{array}$ & $\begin{array}{l}\text { Baseline } \\
\text { BMI } \mathrm{kg} \cdot \mathrm{m}^{-2}\end{array}$ & $\begin{array}{l}\text { Baseline } \\
\text { ESS }\end{array}$ & Design & $\begin{array}{l}\text { CPAP } \\
\text { duration }\end{array}$ & $\begin{array}{c}\text { CPAP } \\
\text { compliance } h \text { per } \\
\text { night } M / F\end{array}$ & Outcomes & $\begin{array}{l}\text { Main findings: } \\
\text { effect of CPAP }\end{array}$ \\
\hline Lemmer [74] & 2016 & OSA & $>30$ & 7 & $60.5 \pm 8.1^{\#}$ & $35.0 \pm 4.7^{\#}$ & $N / R$ & $\begin{array}{l}\text { Baseline to } \\
\text { post-treatment }\end{array}$ & 2 months & $N / R$ & $\begin{array}{c}\text { Wrist } \\
\text { accelerometer }\end{array}$ & $\begin{array}{l}\downarrow \text { sleep movement } \\
\text { arousals after } \\
\text { treatment }\end{array}$ \\
\hline MANSUKHANI [75] & 2012 & OSA & $\bar{x}=16$ & 3 & $44.0 \pm 7.0$ & $N / R$ & $N / R$ & $\begin{array}{l}\text { Baseline to } \\
\text { post-treatment }\end{array}$ & 1 month & $N / R$ & Accelerometer & $\begin{array}{c}\text { Trend toward } \uparrow \\
\text { nonexercise activity } \\
\text { thermogenesis } \\
\text { ( } p=0.08 \text { ) and upright } \\
\text { motion ( } p=0.07 \text { ) after } \\
\text { treatment }\end{array}$ \\
\hline MENDELSON [76] & 2014 & $\begin{array}{l}\text { CPAP, SC } \\
\text { CPAP, TM }\end{array}$ & $\begin{array}{l}>15 \\
>15\end{array}$ & $\begin{array}{l}42 \\
40\end{array}$ & $\begin{array}{l}63.0 \pm 9.0^{\pi} \\
62.0 \pm 9.0^{\circ}\end{array}$ & $\begin{array}{l}30.2 \pm 5.7 n \\
29.6 \pm 3.0^{\pi}\end{array}$ & $\begin{array}{l}8.7 \pm 4.5^{\pi} \\
7.2 \pm 4.3^{\pi}\end{array}$ & $\begin{array}{l}\text { Placebo-controlled, } \\
\text { parallel; RCT }\end{array}$ & 4 months & $\begin{array}{l}4.16 \pm 2.8 \\
3.12 \pm 3.0\end{array}$ & $\begin{array}{l}\text { SenseWear } \\
\text { armband }\end{array}$ & $\begin{array}{l}\leftrightarrow \text { steps per day; } \\
\text { energy expenditure } \\
\text { (kcal) or METS after } \\
\text { treatment in either } \\
\text { group }\end{array}$ \\
\hline PAMIDI [49] & 2015 & $\begin{array}{c}\text { CPAP } \\
\text { Oral placebo }\end{array}$ & $\begin{array}{l}\geqslant 5 \\
\geqslant 5\end{array}$ & $\begin{array}{l}26 \\
13\end{array}$ & $\begin{array}{l}53.8 \pm 6.2 \\
55.2 \pm 8.4\end{array}$ & $\begin{array}{l}36.8 \pm 7.8 \\
32.7 \pm 4.3\end{array}$ & $\begin{array}{l}10.0 \pm 5.9 \\
10.9 \pm 5.0\end{array}$ & $\begin{array}{l}\text { Placebo-controlled, } \\
\text { parallel; RCT }\end{array}$ & $\begin{array}{l}2 \text { weeks } \\
2 \text { weeks }\end{array}$ & 8 & $\begin{array}{c}\text { Wrist } \\
\text { accelerometer }\end{array}$ & $\begin{array}{l}\leftrightarrow \text { physical activity } \\
\text { levels between groups }\end{array}$ \\
\hline TACHIKAWA [48] & 2016 & OSA & $>20$ & 63 & $60.6 \pm 10.0$ & $27.9 \pm 3.8$ & $8.7 \pm 5.3$ & $\begin{array}{l}\text { Baseline to } \\
\text { post-treatment }\end{array}$ & $\begin{array}{l}1 \text { day; } 3 \\
\text { months }\end{array}$ & $4.5 \pm 1.6$ & $\begin{array}{c}\text { Wrist } \\
\text { accelerometer }\end{array}$ & $\begin{array}{l}\leftrightarrow \text { physical activity } \\
\text { levels }\end{array}$ \\
\hline WESt [77] & 2007 & $\begin{array}{l}\text { Active CPAP } \\
\text { Sham CPAP }\end{array}$ & $\begin{array}{l}N / R^{+} \\
N / R^{+}\end{array}$ & $\begin{array}{l}19 \\
21\end{array}$ & $\begin{array}{l}57.8 \pm 10.4 \\
54.5 \pm 9.4\end{array}$ & $\begin{array}{l}36.6 \pm 4.9 \\
36.8 \pm 4.6\end{array}$ & $\begin{array}{l}14.7 \pm 3.5 \\
13.6 \pm 3.5\end{array}$ & $\begin{array}{l}\text { Placebo-controlled, } \\
\text { parallel; RCT }\end{array}$ & 3 months & $\begin{array}{l}3.6 \pm 2.8 \\
3.3 \pm 3.0\end{array}$ & $\begin{array}{c}\text { Wrist } \\
\text { accelerometer }\end{array}$ & $\begin{array}{c}\leftrightarrow \text { physical activity } \\
\text { levels during most } \\
\text { active least active } \\
\text { periods }\end{array}$ \\
\hline WeSt [78] & 2009 & $\begin{array}{l}\text { Active CPAP } \\
\text { Sham CPAP }\end{array}$ & $\begin{array}{l}N / R^{+} \\
N / R^{+}\end{array}$ & $\begin{array}{l}16 \\
20\end{array}$ & $\begin{array}{c}57.2 \pm 11.2 \\
54.1 \pm 9.8\end{array}$ & $\begin{array}{l}37.4 \pm 4.4 \\
36.2 \pm 4.3\end{array}$ & $\begin{array}{l}13.4 \pm 2.6 \\
13.3 \pm 3.4\end{array}$ & $\begin{array}{l}\text { Placebo-controlled, } \\
\text { parallel; RCT }\end{array}$ & 3 months & $\begin{array}{l}3.8 \pm 2.8 \\
3.7 \pm 2.9\end{array}$ & $\begin{array}{c}\text { Wrist } \\
\text { accelerometer }\end{array}$ & $\begin{array}{c}\leftrightarrow \text { mean hourly } \\
\text { physical activity over } \\
24 \mathrm{~h}\end{array}$ \\
\hline $\begin{array}{l}\text { Data are presen } \\
\text { andomised con } \\
\text { elemedicine. } \uparrow: \\
:>10 \text { dips } \cdot h^{-1} \text { in }\end{array}$ & satur & $\begin{array}{l}\text { mean } \pm S D \text {, un } \\
\text { METS: metak }\end{array}$ & ss otherv & $\begin{array}{l}\text { e state } \\
\text { nts; } \mathrm{C} \\
\text { ase. }{ }^{\#} \text { : } \\
\text { ersus F }\end{array}$ & $\begin{array}{l}\text { AHI: apn } \\
\text { conservat } \\
\text { ll OSA grc }\end{array}$ & a-hypopn & $\begin{array}{l}\text { index; } \\
\text { N/R: no } \\
\text { not just } \\
\text { lam withi }\end{array}$ & $\begin{array}{l}\text { M: male; F: female } \\
\text { t reported; MVPA: } \\
\text { t those with physica }\end{array}$ & $\begin{array}{l}\text { e; BMI: bo } \\
\text { moderate } \\
\text { activity } \mathrm{m}\end{array}$ & $\begin{array}{l}\text { dy mass index; } \\
\text {-to-vigorous phys } \\
\text { heasures; " " full g }\end{array}$ & $\begin{array}{l}\text { SS: Epworth Sl } \\
\text { al activity; SC } \\
\text { oups at baselin }\end{array}$ & $\begin{array}{l}\text { eepiness scale; RCT: } \\
\text { standard care; TM: } \\
\text { e, including dropouts; }\end{array}$ \\
\hline
\end{tabular}


accelerometer devices, either at the upper arm, ankle or wrist, may influence recorded activity levels, thereby obscuring results. These differences in methodology should be considered when accounting for reported discrepancies. Furthermore, it has been suggested that combining heart rate monitoring with accelerometry data can improve the precision and accuracy of physical activity assessments [102]. This has not yet been done in the studies of physical activity after CPAP, which suggests a limitation of the current studies and a suggestion for improvement in future investigations.

Findings on physical activity are mixed, probably owing to methodological differences described earlier, as well as study design (pre-post comparisons versus sham-controlled). Some have described an effect of CPAP on improving objectively measured physical activity outcomes, including steps per day [71, 72]. Overall, however, it appears that CPAP has minimal effects on improving free-living physical activity levels. These conclusions are based on subjective, questionnaire-based assessments $[47,70]$ and objective assessments from pre-post comparisons $[34,48]$ and sham-controlled studies [49, 76-78]. The United States Department of Health and Human Services recommends that adults obtain $\geqslant 150$ min of moderate-intensity physical activity or 75 min of vigorous-intensity physical activity each week for optimal health. Therefore, methods to increase physical activity in OSA patients have the potential to not only reduce body weight, but also to improve cardiovascular health and fitness [103]. Efforts should be made by clinicians to promote lifestyle modifications to increase physical activity levels in addition to CPAP use.

\section{Future directions}

Future studies should include RCTs on topics that expand on those described here. For example, recent work suggests an effect of CPAP on circulating levels of glucagon-like protein-1, a satiety hormone [104]. Interestingly, an animal model of OSA found that induced intermittent hypoxia in mice caused alterations in microbiota composition and diversity [105], suggesting the testing of CPAP on this outcome. Based on findings that sleep restriction can affect brain regions involved in motivation and reward in the context of food stimuli [106], new work should also extend beyond the hormonal/homeostatic control of energy intake, to determine how CPAP affects the hedonic and cognitive control of food intake in OSA patients, using functional brain imaging.

\section{Conclusions}

This article describes the current state of the literature on energy balance regulation in response to CPAP in OSA patients. Based on a systematic review of the literature, it is not yet fully apparent whether CPAP has an appreciable effect on influencing energy expenditure and energy intake to promote changes in energy balance. Yet the question remains as to why alleviating the symptoms associated with OSA is sometimes associated with changes in body weight. Indeed, a recent meta-analysis of RCTs indicates that CPAP treatment actually promotes weight gain [37]. The regulation of energy balance in OSA is complex and multifactorial, involving food intake, hormonal regulation of hunger and satiety and energy expenditure via metabolism and free-living physical activity. As has been described, it appears that CPAP may reduce energy expenditure via metabolism but that it does not induce a compensatory increase in physical activity to offset these reductions. Furthermore, although it may alter hormones in a way expected to reduce hunger and food intake, it remains to be seen whether energy intake is in fact reduced by CPAP. Thus, the full picture of energy balance regulation in response to CPAP is still incomplete. Understanding how the components of energy balance are affected by CPAP will allow clinicians to more effectively guide overall treatment approaches to optimise weight loss and symptom amelioration in OSA patients. Promoting CPAP in conjunction with other weight loss approaches, such as intensive lifestyle intervention, could be used to encourage optimal outcomes for weight management in OSA patients.

\section{References}

1 Schäfer H, Pauleit D, Sudhop T, et al. Body fat distribution, serum leptin, and cardiovascular risk factors in men with obstructive sleep apnea. Chest 2002; 122: 829-839.

2 Oğretmenoğlu O, Süslü AE, Yücel OT, et al. Body fat composition: a predictive factor for obstructive sleep apnea. Laryngoscope 2005; 115: 1493-1498.

3 Lovin S, Bercea R, Cojocaru C, et al. Body composition in obstructive sleep apneahypopnea syndrome bio-impedance reflects the severity of sleep apnea. Multidiscip Respir Med 2010; 5: 44-49.

4 Shinohara E, Kihara S, Yamashita S, et al. Visceral fat accumulation as an important risk factor for obstructive sleep apnoea syndrome in obese subjects. J Intern Med 1997; 241: 11-18.

5 Romero-Corral A, Caples SM, Lopez-Jimenez F, et al. Interactions between obesity and obstructive sleep apnea: implications for treatment. Chest 2010; 137: 711-719.

6 St-Onge MP, Shechter A. Sleep disturbances, body fat distribution, food intake and/or energy expenditure: pathophysiological aspects. Horm Mol Biol Clin Investig 2014; 17: 29-37.

7 Barceló A, Barbé F, Llompart E, et al. Neuropeptide Y and leptin in patients with obstructive sleep apnea syndrome: role of obesity. Am J Respir Crit Care Med 2005; 171: 183-187.

8 Basoglu OK, Sarac F, Sarac S, et al. Metabolic syndrome, insulin resistance, fibrinogen, homocysteine, leptin, and C-reactive protein in obese patients with obstructive sleep apnea syndrome. Ann Thorac Med 2011; 6: 120-125. 
Harsch IA, Konturek PC, Koebnick C, et al. Leptin and ghrelin levels in patients with obstructive sleep apnoea: effect of CPAP treatment. Eur Respir J 2003; 22: 251-257.

Ip MS, Lam KS, Ho C, et al. Serum leptin and vascular risk factors in obstructive sleep apnea. Chest 2000; 118: 580-586.

Kapsimalis F, Varouchakis G, Manousaki A, et al. Association of sleep apnea severity and obesity with insulin resistance, C-reactive protein, and leptin levels in male patients with obstructive sleep apnea. Lung 2008; 186: 209-217.

Ozturk L, Unal M, Tamer L, et al. The association of the severity of obstructive sleep apnea with plasma leptin levels. Arch Otolaryngol Head Neck Surg 2003; 129: 538-540.

Papaioannou I, Patterson M, Twigg GL, et al. Lack of association between impaired glucose tolerance and appetite regulating hormones in patients with obstructive sleep apnea. J Clin Sleep Med 2011; 7: 486-492.

Phillips BG, Kato M, Narkiewicz K, et al. Increases in leptin levels, sympathetic drive, and weight gain in obstructive sleep apnea. Am J Physiol Heart Circ Physiol 2000; 279: H234-H237.

Ulukavak Ciftci T, Kokturk O, Bukan N, et al. Leptin and ghrelin levels in patients with obstructive sleep apnea syndrome. Respiration 2005; 72: 395-401.

Vgontzas AN, Papanicolaou DA, Bixler EO, et al. Sleep apnea and daytime sleepiness and fatigue: relation to visceral obesity, insulin resistance, and hypercytokinemia. J Clin Endocrinol Metab 2000; 85: 1151-1158.

Chihara Y, Akamizu T, Azuma M, et al. Among metabolic factors, significance of fasting and postprandial increases in acyl and desacyl ghrelin and the acyl/desacyl ratio in obstructive sleep apnea before and after treatment. J Clin Sleep Med 2015; 11: 895-905.

Takahashi K, Chin K, Akamizu T, et al. Acylated ghrelin level in patients with OSA before and after nasal CPAP treatment. Respirology 2008; 13: 810-816.

Ursavas A, Ilcol YO, Nalci N, et al. Ghrelin, leptin, adiponectin, and resistin levels in sleep apnea syndrome: role of obesity. Ann Thorac Med 2010; 5: 161-165.

Beebe DW, Miller N, Kirk S, et al. The association between obstructive sleep apnea and dietary choices among obese individuals during middle to late childhood. Sleep Med 2011; 12: 797-799.

Smith SS, Waight C, Doyle G, et al. Liking for high fat foods in patients with obstructive sleep apnoea. Appetite 2014; 78: 185-192.

Quan SF, O'Connor GT, Quan JS, et al. Association of physical activity with sleep-disordered breathing. Sleep Breath 2007; 11: 149-157.

Chasens ER, Sereika SM, Houze MP, et al. Subjective and objective appraisal of activity in adults with obstructive sleep apnea. J Aging Res 2011; 2011: 751819.

Ucok K, Aycicek A, Sezer M, et al. Resting metabolic rate and anthropometric measurements in male sleep apnea patients. Intern Med 2011; 50: 833-838.

Lin CC, Chang KC, Lee KS. Effects of treatment by laser-assisted uvuloplasty on sleep energy expenditure in obstructive sleep apnea patients. Metabolism 2002; 51: 622-627.

Stenlöf K, Grunstein R, Hedner J, et al. Energy expenditure in obstructive sleep apnea: effects of treatment with continuous positive airway pressure. Am J Physiol 1996; 271: E1036-E1043.

Peppard PE, Young T, Palta M, et al. Longitudinal study of moderate weight change and sleep-disordered breathing. JAMA 2000; 284: 3015-3021.

Tuomilehto HP, Seppa JM, Partinen MM, et al. Lifestyle intervention with weight reduction: first-line treatment in mild obstructive sleep apnea. Am J Respir Crit Care Med 2009; 179: 320-327.

Johansson K, Neovius M, Lagerros YT, et al. Effect of a very low energy diet on moderate and severe obstructive sleep apnoea in obese men: a randomised controlled trial. BMJ 2009; 339: b4609.

Foster GD, Borradaile KE, Sanders MH, et al. A randomized study on the effect of weight loss on obstructive sleep apnea among obese patients with type 2 diabetes: the Sleep AHEAD study. Arch Intern Med 2009; 169: 1619-1626.

Loube DI, Loube AA, Erman MK. Continuous positive airway pressure treatment results in weight loss in obese and overweight patients with obstructive sleep apnea. J Am Diet Assoc 1997; 97: 896-897.

Chin K, Shimizu K, Nakamura T, et al. Changes in intra-abdominal visceral fat and serum leptin levels in patients with obstructive sleep apnea syndrome following nasal continuous positive airway pressure therapy. Circulation 1999; 100: 706-712.

Cuhadaroğlu C, Utkusavas A, Oztürk L, et al. Effects of nasal CPAP treatment on insulin resistance, lipid profile, and plasma leptin in sleep apnea. Lung 2009; 187: 75-81.

Diamanti C, Manali E, Ginieri-Coccossis M, et al. Depression, physical activity, energy consumption, and quality of life in OSA patients before and after CPAP treatment. Sleep Breath 2013; 17: 1159-1168.

Quan SF, Budhiraja R, Clarke DP, et al. Impact of treatment with continuous positive airway pressure (CPAP) on weight in obstructive sleep apnea. J Clin Sleep Med 2013; 9: 989-993.

Redenius R, Murphy C, O'Neill E, et al. Does CPAP lead to change in BMI? J Clin Sleep Med 2008; 4: 205-209.

Drager LF, Brunoni AR, Jenner R, et al. Effects of CPAP on body weight in patients with obstructive sleep apnoea: a meta-analysis of randomised trials. Thorax 2015; 70: 258-264.

Igelström H, Emtner M, Lindberg E, et al. Tailored behavioral medicine intervention for enhanced physical activity and healthy eating in patients with obstructive sleep apnea syndrome and overweight. Sleep Breath 2014; 18: 655-668.

Lee P, Yu CW, Lin MT, et al. CPAP effects on leptin and visceral fat in patients with sleep apnea: double-blind, randomized, controlled trial. Eur Respir J 2011; 38: Suppl. 55, 1428.

McCullough PA, Speck BJ, Winslow DH, et al. Sleep/wake activity and energy expenditure in overweight and obese obstructive sleep apnea patients: a pre- and post-CPAP comparison. Sleep 2012; 35: A177.

Mendelson M, Vivodtzev I, Taminiser R, et al. Continuous positive airway pressure (CPAP) supported by telemedicine improves sleepiness and quality of life but not blood pressure in high cardiovascular risk obstructive sleep apnea (OSA): a randomized, controlled trial. Eur Respir J 2013; 42: Suppl. 57, P2044.

Pepin JL, Mendelson M, Vivodtzev I, et al. Continuous positive airway pressure (CPAP) supported by telemedicine improves sleepiness and quality of life but not blood pressure in high cardiovascular risk obstructive sleep apnea (OSA): a randomized, controlled trial. Am J Respir Crit Care Med 2014; 189: A5626. 

obstructive sleep apnea on physical activity and glucose control in adults with type 2 diabetes: results of a pilot study. Am J Respir Crit Care Med 2012; 185: A2164.

44 Fekete K, Boutou A, Pitsiou G, et al. Resting energy expenditure among patients with OSAS: the impact of a single CPAP application. Eur Respir J 2015; 46: Suppl. 59, PA4171.

45 Tachikawa R, Ikeda K, Matsumoto T, et al. Effects of CPAP on energy balance in patients with obstructive sleep apnea. Eur Respir J 2015; 46: Suppl. 59, PA2371.

46 Bruyneel M, Sanida C, Doyen C, et al. Sleep duration is increased but not physical activity in somnolent moderate to severe obstructive sleep apnea patients treated by continuous positive airway pressure. Somnologie 2015; 19: 38 .

47 Batool-Anwar S, Goodwin JL, Drescher AA, et al. Impact of CPAP on activity patterns and diet in patients with obstructive sleep apnea (OSA). J Clin Sleep Med 2014; 10: 465-472.

48 Tachikawa R, Ikeda K, Minami T, et al. Changes in energy metabolism after continuous positive airway pressure for obstructive sleep apnea. Am J Respir Crit Care Med 2016; 194: 729-738 .

49 Pamidi S, Wroblewski K, Stepien M, et al. Eight hours of nightly continuous positive airway pressure treatment of obstructive sleep apnea improves glucose metabolism in patients with prediabetes. A randomized controlled trial. Am J Respir Crit Care Med 2015; 192: 96-105.

50 Chin K, Nakamura T, Takahashi K, et al. Effects of obstructive sleep apnea syndrome on serum aminotransferase levels in obese patients. Am J Med 2003; 114: 370-376.

51 Harsch IA, Wallaschofski H, Koebnick C, et al. Adiponectin in patients with obstructive sleep apnea syndrome: course and physiological relevance. Respiration 2004; 71: 580-586.

52 Sanner BM, Kollhosser P, Buechner N, et al. Influence of treatment on leptin levels in patients with obstructive sleep apnoea. Eur Respir J 2004; 23: 601-604.

53 Shimizu K, Chin K, Nakamura T, et al. Plasma leptin levels and cardiac sympathetic function in patients with obstructive sleep apnoea-hypopnoea syndrome. Thorax 2002; 57: 429-434.

54 Harsch IA, Schahin SP, Radespiel-Tröger M, et al. Continuous positive airway pressure treatment rapidly improves insulin sensitivity in patients with obstructive sleep apnea syndrome. Am J Respir Crit Care Med 2004; 169: 156-162.

Saarelainen S, Lahtela J, Kallonen E. Effect of nasal CPAP treatment on insulin sensitivity and plasma leptin. J Sleep Res 1997; 6: 146-147.

56 Trenell MI, Ward JA, Yee BJ, et al. Influence of constant positive airway pressure therapy on lipid storage, muscle metabolism and insulin action in obese patients with severe obstructive sleep apnoea syndrome. Diabetes Obes Metab 2007; 9: 679-687.

57 Drummond M, Winck JC, Guimarães JT, et al. Autoadjusting-CPAP effect on serum leptin concentrations in obstructive sleep apnoea patients. BMC Pulm Med 2008; 8: 21.

58 Garcia JM, Sharafkhaneh H, Hirshkowitz M, et al. Weight and metabolic effects of CPAP in obstructive sleep apnea patients with obesity. Respir Res 2011; 12: 80.

59 Murri M, Alcázar-Ramírez J, Garrido-Sánchez L, et al. Oxidative stress and metabolic changes after continuous positive airway pressure treatment according to previous metabolic disorders in sleep apnea-hypopnea syndrome patients. Transl Res 2009; 154: 111-121.

60 Kritikou I, Basta M, Vgontzas AN, et al. Sleep apnoea, sleepiness, inflammation and insulin resistance in middle-aged males and females. Eur Respir J 2014; 43: 145-155.

61 Hoyos CM, Killick R, Yee BJ, et al. Cardiometabolic changes after continuous positive airway pressure for obstructive sleep apnoea: a randomised sham-controlled study. Thorax 2012; 67: 1081-1089.

62 Busquets X, Barbé F, Barceló A, et al. Decreased plasma levels of orexin-A in sleep apnea. Respiration 2004; 71: 575-579.

63 Igarashi N, Tatsumi K, Nakamura A, et al. Plasma orexin-A levels in obstructive sleep apnea-hypopnea syndrome. Chest 2003; 124: 1381-1385.

64 Sakurai S, Nishijima T, Takahashi S, et al. Low plasma orexin-A levels were improved by continuous positive airway pressure treatment in patients with severe obstructive sleep apnea-hypopnea syndrome. Chest 2005; 127: 731-737.

65 Fekete K, Boutou AK, Pitsiou G, et al. Resting energy expenditure in OSAS: the impact of a single CPAP application. Sleep Breath 2016; 20: 121-128.

66 Ryan CF, Love LL, Buckley PA. Energy expenditure in obstructive sleep apnea. Sleep 1995; 18: $180-187$.

67 Smurra M, Philip P, Taillard J, et al. CPAP treatment does not affect glucose-insulin metabolism in sleep apneic patients. Sleep Med 2001; 2: 207-213.

68 Shechter A, Pham T, Rising R, et al. Effects of CPAP on energy expenditure in obese obstructive sleep apnoea patients: a pilot study. Obes Res Clin Pract 2015; 9: 618-621.

69 Billings CG, Aung T, Renshaw SA, et al. Incremental shuttle walk test in the assessment of patients with obstructive sleep apnea-hypopnea syndrome. J Sleep Res 2013; 22: 471-477.

70 Salord N, Fortuna AM, Monasterio C, et al. A randomized controlled trial of continuous positive airway pressure on glucose tolerance in obese patients with obstructive sleep apnea. Sleep 2016; 39: 35-41.

71 Chasens ER, Korytkowski M, Sereika SM, et al. Improving activity in adults with diabetes and coexisting obstructive sleep apnea. West J Nurs Res 2014; 36: 294-311.

72 Jean R. Improvement in physical activity in patients with obstructive sleep apnea (OSA) treated with continuous positive airway pressure. Sleep 2015; 38: A182-A183.

73 Kerley C, Bolger K, Fennell K, et al. Influence of continuous positive airway pressure therapy on body composition and total energy expenditure in obstructive sleep apnoea syndrome. Ir J Med Sci 2012; 181: S407-S408.

74 Lemmer B, Scholtze J, Schmitt J. Circadian rhythms in blood pressure, heart rate, hormones, and on polysomnographic parameters in severe obstructive sleep apnea syndrome patients: effect of continuous positive airway pressure. Blood Press Monit 2016; 21: 136-143.

75 Mansukhani MP, McCrady-Spitzer S, Levine J, et al. Non-exercise activity thermogenesis (NEAT) in obstructive sleep apnea: a pilot study. Sleep 2012; 35: A136. 

1863-1870. activity in patients with obstructive sleep apnoea: a randomised controlled trial. Sleep Med 2009; 10: 1056-1058. sleep apnoea and type 2 diabetes. Thorax 2007; 62: 969-974.

79 Vasquez MM, Goodwin JL, Drescher AA, et al. Associations of dietary intake and physical activity with sleep disordered breathing in the Apnea Positive Pressure Long-Term Efficacy Study (APPLES). J Clin Sleep Med 2008; 4: 411-418.

80 Pamidi S, Stepien M, Sharif-Sidi K, et al. Effective treatment of obstructive sleep apnea improves glucose tolerance in prediabetes: a randomized placebo-controlled study. Am J Respir Crit Care Med 2013; 187: A2381.

81 Dhurandhar NV, Schoeller D, Brown AW, et al. Energy balance measurement: when something is not better than nothing. Int J Obes 2015; 39: 1109-1113.

82 Muhlheim LS, Allison DB, Heshka S, et al. Do unsuccessful dieters intentionally underreport food intake? Int J Eat Disord 1998; 24: 259-266.

de Castro JM. Eating behavior: lessons from the real world of humans. Nutrition 2000; 16: 800-813.

Zhang F, Chen Y, Heiman M, et al. Leptin: structure, function and biology. Vitam Horm 2005; 71: 345-372.

Iftikhar IH, Hoyos CM, Phillips CL, et al. Meta-analyses of the association of sleep apnea with insulin resistance, and the effects of CPAP on HOMA-IR, adiponectin, and visceral adipose fat. J Clin Sleep Med 2015; 11: 475-485. Campo A, Frühbeck G, Zulueta JJ, et al. Hyperleptinaemia, respiratory drive and hypercapnic response in obese patients. Eur Respir J 2007; 30: 223-231.

87 Grosfeld A, Zilberfarb V, Turban S, et al. Hypoxia increases leptin expression in human PAZ6 adipose cells. Diabetologia 2002; 45: 527-530.

88 Tatsumi K, Kasahara Y, Kurosu K, et al. Sleep oxygen desaturation and circulating leptin in obstructive sleep apnea-hypopnea syndrome. Chest 2005; 127: 716-721.

89 Shea SA, Hilton MF, Orlova C, et al. Independent circadian and sleep/wake regulation of adipokines and glucose in humans. J Clin Endocrinol Metab 2005; 90: 2537-2544.

90 Simon C, Gronfier C, Schlienger JL, et al. Circadian and ultradian variations of leptin in normal man under continuous enteral nutrition: relationship to sleep and body temperature. J Clin Endocrinol Metab 1998; 83: 1893-1899.

91 Fenzl T, Flachskamm C, Rossbauer M, et al. Circadian rhythms of basal orexin levels in the hypothalamus are not influenced by an impaired corticotropin-releasing hormone receptor type 1 system. Behav Brain Res 2009; 203: $143-145$.

92 Natalucci G, Riedl S, Gleiss A, et al. Spontaneous 24-h ghrelin secretion pattern in fasting subjects: maintenance of a meal-related pattern. Eur J Endocrinol 2005; 152: 845-850.

93 Dallongeville J, Hecquet B, Lebel P, et al. Short term response of circulating leptin to feeding and fasting in man: influence of circadian cycle. Int J Obes Relat Metab Disord 1998; 22: 728-733.

94 Cummings DE, Purnell JQ, Frayo RS, et al. A preprandial rise in plasma ghrelin levels suggests a role in meal initiation in humans. Diabetes 2001; 50: 1714-1719.

95 Illner K, Brinkmann G, Heller M, et al. Metabolically active components of fat free mass and resting energy expenditure in nonobese adults. Am J Physiol Endocrinol Metab 2000; 278: E308-E315.

96 Stenlöf K, Grunstein R, Hedner J, et al. Energy expenditure in obstructive sleep apnea: effects of treatment with continuous positive airway pressure. Am I Physiol 1996; 271: E1036-E1043.

97 Rodway GW, Weaver TE, Mancini C, et al. Evaluation of sham-CPAP as a placebo in CPAP intervention studies. Sleep 2010; 33: 260-266.

98 Hill JO, Wyatt HR, Peters JC. Energy balance and obesity. Circulation 2012; 126: 126-132.

99 St-Onge MP, Roberts AL, Chen J, et al. Short sleep duration increases energy intakes but does not change energy expenditure in normal-weight individuals. Am J Clin Nutr 2011; 94: 410-416.

100 Nedeltcheva AV, Kilkus JM, Imperial J, et al. Sleep curtailment is accompanied by increased intake of calories from snacks. Am J Clin Nutr 2009; 89: 126-133.

101 Seale JL, Rumpler WV, Conway JM, et al. Comparison of doubly labeled water, intake-balance, and direct- and indirect-calorimetry methods for measuring energy expenditure in adult men. Am J Clin Nutr 1990; 52: 66-71.

102 Brage S, Ekelund U, Brage N, et al. Hierarchy of individual calibration levels for heart rate and accelerometry to measure physical activity. I Appl Physiol 2007; 103: 682-692.

103 Bouchard C, Blair SN, Katzmarzyk PT. Less sitting, more physical activity, or higher fitness? Mayo Clin Proc 2015; 90: 1533-1540.

104 Matsumoto T, Harada N, Azuma M, et al. Plasma incretin levels and dipeptidyl peptidase-4 activity in patients with obstructive sleep apnea. Ann Am Thorac Soc 2016; 13: 1378-1387.

105 Moreno-Indias I, Torres M, Montserrat JM, et al. Intermittent hypoxia alters gut microbiota diversity in a mouse model of sleep apnoea. Eur Respir J 2015; 45: 1055-1065.

106 St-Onge MP, Wolfe S, Sy M, et al. Sleep restriction increases the neuronal response to unhealthy food in normal-weight individuals. Int J Obes 2013; 38: 411-416. 